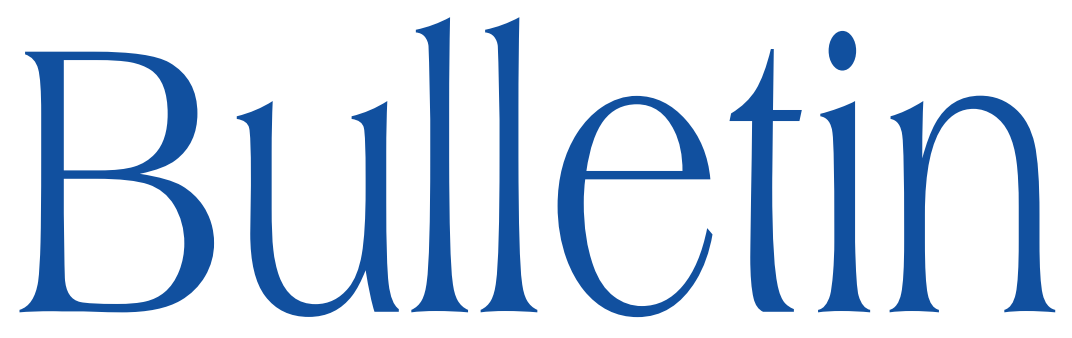

de la SOCIÉTÉ MATHÉMATIQUE DE FRANCE

\title{
STRATIFIED WHITNEY JETS AND TEMPERED ULTRADISTRIBUTIONS ON THE SUBANALYTIC SITE
}

\author{
N. Honda \& G. Morando
}

\section{Tome 139}

Fascicule 3

2011 


\title{
STRATIFIED WHITNEY JETS AND TEMPERED ULTRADISTRIBUTIONS ON THE SUBANALYTIC SITE
}

\author{
By N. Honda \& G. Morando
}

\begin{abstract}
In this paper we introduce the sheaf of stratified Whitney jets of Gevrey order on the subanalytic site relative to a real analytic manifold $X$. Then, we define stratified ultradistributions of Beurling and Roumieu type on $X$. In the end, by means of stratified ultradistributions, we define tempered-stratified ultradistributions and we prove two results. First, if $X$ is a real surface, the tempered-stratified ultradistributions define a sheaf on the subanalytic site relative to $X$. Second, the tempered-stratified ultradistributions on the complementary of a 1-regular closed subset of $X$ coincide with the sections of the presheaf of tempered ultradistributions.
\end{abstract}

RÉsumé (Jets stratifiés de Whitney et ultradistributions tempérées sur le site sousanalytique)

Dans cet article nous introduisons le faisceau des jets de Whitney d'ordre de Gevrey sur le site sous-analytique relatif à une variété analytique réelle $X$. Ensuite, nous définissons les ultradistributions tempérées sur $X$ de type Beurling et Roumieu. Enfin, à travers les ultradistributions stratifiées, nous définissons les ultradistributions tempérées-stratifiées et nous démontrons les deux résultats suivants : (a) si $X$ est une

Texte reçu le 11 novembre 2009, révisé le 4 février 2010 et le 11 février 2010, accepté le 19 février 2010.

N. Honda, Department of Mathematics, Faculty of Science, Hokkaido University Kita 10, Nishi 8, Kita-Ku, Sapporo, Hokkaido, 060-0810, Japan

E-mail : honda@math.sci.hokudai.ac.jp

G. Morando, Dipartimento di Matematica Pura ed Applicata, Università di Padova, Via Trieste 63, 35121 Padova, Italy, and Centro de Álgebra da Universidade de Lisboa, Av. Prof. Gama Pinto, 2, 1649-003 Lisboa, Portugal. - E-mail : gmorando@math.unipd.it

2000 Mathematics Subject Classification. - 46M20; 46F05 32B20 32C38.

Key words and phrases. - Sheaves on subanalytic sites, tempered ultradistributions, Whitney jets.

The first author has been supported by Grant-in-Aid for Scientific Research, No. 20234567. The second author has been supported in part by grant CPDA061823 of Padova University. 
surface réelle, les ultradistributions tempérées-stratifiées définissent un faisceau sur le site sous-analytique relatif à $X$, et (b) les ultradistributions tempérées-stratifiées sur le complémentaire d'un sous-ensemble fermé 1-régulier de $X$, coïncident avec les sections du préfaisceau des ultradistributions tempérées.

\section{Introduction}

One of the aims of the present article is to define tempered ultradistributions of Beurling and Roumieu class of order $s>1$ and Whitney jets with growth conditions as sheaves on the subanalytic site relative to a real analytic manifold $X$. As growth conditions are not of local nature, functional spaces defined on open subsets of $X$, as tempered distributions, Whitney $\mathscr{C}^{\infty}$-functions or holomorphic functions with polynomial growth at the boundary do not glue on arbitrary coverings. In particular, such spaces do not define sheaves on the usual topology of an analytic manifold. We recall the approach set by S. Łojasiewicz ([12]) later reinterpreted and generalized in the works of $M$. Kashiwara and P. Schapira (see [4], [6] and [7]). They defined tempered distributions and Whitney $\mathscr{C}^{\infty}$-functions as sheaves on the subanalytic site, $X_{\mathrm{sa}}$, relative to a real analytic manifold $X$. The open sets of $X_{\text {sa }}$ are the relatively compact subanalytic open subsets of $X$ and the coverings are those admitting finite refinements. The use of these objects in the study of linear ordinary differential equations gave interesting results (see [14]). Let us mention that function spaces with growth conditions, such as holomorphic functions on the complex plane with moderate or Gevrey growth or asymptotic expansion at the origin, are treated as sheaves on the real blow up at the origin by B. Malgrange in [13] and many other authors elsewhere in litterature. Such function spaces are used in a systematic way in the study of linear ordinary differential equations. Some of these sheaves on the real blow up at the origin can be obtained by specializing their subanalytic generalization (see [15]).

Among the motivations of this paper there is the fact that the naive definition of tempered ultradistributions, mimicking that of tempered distributions (see [4]), does not give a sheaf on the subanalytic site, as explained in Section 1.3. Let us recall that tempered ultradistributions on an open set $U$ in $X$ are defined as global sections of ultradistributions modulo ultradistributions with support on $X \backslash U$. This latter space is the dual of Whitney jets with Gevrey like growth conditions on $X \backslash U$. In this paper, we relax the condition on Whitney jets with Gevrey like growth conditions by introducing the stratified Whitney jets on a real analytic manifold $X$. We prove decomposition and gluing properties for stratified Whitney jets on finitely many subanalytic subsets of $X$ (Lemma 2.2.4). Then we study the dual of stratified Whitney jets

TOME $139-2011-\mathrm{N}^{\mathrm{O}} 3$ 
on a closed set $Z \subset X$, the space of stratified ultradistributions on $Z$. This latter space is a subspace of ultradistributions with support in $Z$. We study the decomposability of stratified ultradistributions on arbitrary finitely many subanalytic closed sets (Corollary 2.4.5 and Corollary 3.1.5). Then we define tempered-stratified ultradistributions on $U$ as global ultradistributions modulo stratified ultradistributions on $X \backslash U$. We prove that, when $X$ has dimension 2, tempered-stratified ultradistributions define a sheaf on $X_{\mathrm{sa}}$. Further, we prove that, if $X \backslash U$ satisfies a regularity condition, tempered-stratified ultradistributions on $U$ coincide with classical tempered ultradistributions on $U$ (Theorem 3.2.1). We conclude by proving that tempered-stratified ultradistributions and other spaces of ultradistributions similarly defined do not give rise to sheaves on $X_{\mathrm{sa}}$, if $X$ has dimension $>2$.

Similar results on the decomposability of ultradistributions were obtained by J.-M. Kantor ([3]) and by A. Lambert ([11]). Their approach is quite different from our. Indeed, given $s>1$, they find a family $\mathcal{T}_{s}$ of subanalytic closed sets depending on $s$ such that ultradistributions of class $s$ decompose on sets in $\mathcal{T}_{s}$. The family $\mathcal{T}_{s}$ is not closed under intersections hence it is not possible to define a Grothendieck topology and a notion of sheaf starting from it.

In the end, let us recall that ultradistributions and growth conditions of Gevrey type turned out to be very useful in the functorial study of linear diffential equations, being strictly linked to the irregularity of equations. Let us cite, for example, [2] and [19] for some applications of ultradistributions in the study of systems of linear differential equations. In the present article we do not use tempered-stratified ultradistributions to study systems of linear differential equations, postponing this problem to future investigations. Throughout the paper, we just limit to point out if the sheaves we define give rise to sheaves of modules over the ring of linear differential operators with analytic coefficients.

The paper is organized as follows. We start Section 1 by recalling the basic properties of Whitney jets with growth conditions. Then, mimicking [4], we define the presheaf of tempered ultradistributions and we recall a condition, due to H. Komatsu, for a continuous function to extend to the whole space as an ultradistribution. In the end of the section, we prove that tempered ultradistributions do not glue on finitely many subanalytic open subsets of $\mathbb{R}^{2}$.

In Section 2 we start by recalling some definitions and basic results on subanalytic sets and the subanalytic site relative to a real analytic manifold $X$. Then, we define the space of stratified Whitney jets with Gevrey growth conditions and we prove that they give rise to a sheaf on the subanalytic site relative to $X$. Then, we introduce the space of stratified ultradistributions on $X$ and we prove that this space is dual to stratified Whitney jets. In the end of the section, from the gluing property of stratified Whitney jets, we obtain a decomposition property for stratified ultradistributions. 
In Section 3, given a real analytic manifold $X$, we define tempered-stratified ultradistributions on a subanalytic open set $U \subset X$ which is a subspace of tempered ultradistributions on $U$. Then, we prove two results. The first states that, if $\operatorname{dim} X=2$, tempered-stratified ultradistributions define a sheaf on the subanalytic site relative to $X$. The second states that if $X \backslash U$ satisfies a regularity condition, then tempered-stratified ultradistributions on $U$ coincide with tempered ultradistributions on $U$.

In Appendix A we prove a result of density for stratified Whitney jets in the space of Whitney jets. Such result is needed in Section 2, we prove it in the Appendix as the proof is rather long and technical.

Acknowledgements. - During the preparation of this article, the second author has benefited of a JSPS Summer Program scholarship and of a scholarship from the Fundação para a Ciência e a Tecnologia at the Centro de Álgebra da Universidade de Lisboa. The second author would like to thank JSPS, FCT and CAUL for their support.

\section{Notations and review on Whitney jets and ultradistributions}

In this paper, we assume that a real analytic manifold is countable at infinity.

1.1. Whitney jets with Gevrey conditions. - Let $X$ be a real analytic manifold. We denote by $\operatorname{Mod}\left(\mathbb{C}_{X}\right)$ the category of sheaves on $X$ with values in $\mathbb{C}$-vector spaces, and by $\mathscr{C}^{\infty}$ the sheaf of infinitely differentiable functions on $X$. We denote by $\pi_{k}: J^{k} \rightarrow X\left(k \in \mathbb{Z}_{\geq 0}\right)$ the vector bundle associated with $\mathrm{k}$-th jets over $X$. For any non-negative integers $k_{1} \geq k_{2}$, the morphism of vector bundles $j^{k_{2}, k_{1}}: J^{k_{1}} \rightarrow J^{k_{2}}$ is defined by the canonical projection from $k_{1}$-th jets to $k_{2}$-th jets.

Let $A$ be a locally closed subset in $X$, and $J^{k}(A)$ designates the set of continuous sections of the vector bundle $J^{k}$ over $A$. We denote by $j_{X}^{k}: \mathscr{C}^{\infty}(X) \rightarrow$ $J^{k}(X)$ the canonical jets extension morphism, and for any locally closed spaces $A \subset B$, we designate by $j_{A, B}^{k}: J^{k}(B) \rightarrow J^{k}(A)$ the natural restriction map from sections over $B$ to those over $A$. Composing $j_{X}^{k}$ and $j_{A, X}^{k}$ we have the canonical morphism

$$
j_{A}^{k}=j_{A, X}^{k} \circ j_{X}^{k}: \mathscr{C}^{\infty}(X) \rightarrow J^{k}(A) .
$$

The morphism of vector bundles $j^{k_{2}, k_{1}}\left(k_{1} \geq k_{2}\right)$ induces the map

$$
j_{A}^{k_{2}, k_{1}}: J^{k_{1}}(A) \rightarrow J^{k_{2}}(A)
$$

TOME $139-2011-\mathrm{N}^{\mathrm{O}} 3$ 
and using these maps we define the jets space over $A$ by

$$
J(A):=\lim _{k \in \mathbb{Z}_{\geq 0}} J^{k}(A)
$$

The morphism $j_{X}^{k}$ (resp. $j_{A, B}^{k}$ ) induces

$$
\begin{aligned}
& j_{X}: \mathscr{C}^{\infty}(X) \rightarrow J(X) \\
&\text { (resp. } \left.\quad j_{A, B}: J(B) \rightarrow J(A)\right) .
\end{aligned}
$$

We set $j_{A}:=j_{A, X} \circ j_{X}: \mathscr{C}^{\infty}(X) \rightarrow J(A)$.

If $X=\mathbb{R}^{n}$ with a system of coordinates $\left(x_{1}, x_{2}, \ldots, x_{n}\right)$, then the jets space $J(A)$ is isomorphic to the set

$$
\left\{\left\{f_{\alpha}\right\}_{\alpha \in\left(\mathbb{Z}_{\geq 0}\right)^{n}} ; f_{\alpha} \in \mathscr{C}^{0}(A)\right\}
$$

where $\mathscr{C}^{0}(A)$ designates the set of continuous functions on $A$. The map $j_{A}$ is identified with

$$
j_{A}(f)=\left\{\left.\frac{\partial^{\alpha} f}{\partial x^{\alpha}}\right|_{A}\right\}_{\alpha \in\left(\mathbb{Z}_{\geq 0}\right)^{n}} \quad \text { for } f \in \mathscr{C}^{\infty}(X) .
$$

Let $A$ be a locally closed subset in $X$. We define $\mathscr{J}_{A} \in \operatorname{Mod}\left(\mathbb{C}_{X}\right)$ by

$$
\mathscr{J}_{A}(U):=J(A \cap U)
$$

for $U$ an open subset of $X$. If $A \cap U=\varnothing$, then we consider $J(A \cap U)$ as the zero object. The morphism $j_{A \cap U, U} \circ j_{U}: \mathscr{C}^{\infty}(U) \rightarrow J(A \cap U)$ induces the sheaf homomorphism $j_{A}: \mathscr{C}^{\infty} \rightarrow \mathcal{J}_{A}$.

We have that $\mathscr{J}_{A}$ is a sheaf of rings and modules over $\mathscr{D}_{X}$ : the sheaf of rings of linear differential operators with analytic coefficients on $X$.

From now on, the symbol $*$ denotes $(s)$ or $\{s\}$ for some $s>1$. Let us recall the definition of the sheaf $\mathscr{C}^{*}$ of ultra-differentiable functions of class $*$ in $X$.

First we need the notion of 1-regular sets.

Definition 1.1.1. - We say that $A$ is 1-regular at $p \in X$ if there exist a neighborhood $U \subset X$ of $p$, a neighborhood $V \subset \mathbb{R}^{n}$ of the origin and an isomorphism $\psi:(U, p) \rightarrow(V, 0)$ satisfying the following condition. There exist a positive constant $\kappa>0$ and a compact neighborhood $K \subset V$ of the origin such that for any $x_{1}, x_{2} \in \psi(A \cap U) \cap K$ there exists a subanalytic curve $l$ in $\psi(A \cap U)$ joining $x_{1}$ and $x_{2}$ and satisfying the estimate

$$
|l| \leq \kappa\left|x_{1}-x_{2}\right|
$$

where $|l|$ stands for the length of $l$.

The set $A$ is said to be 1-regular if it is 1-regular at any point $p \in X$. 
For a locally closed subanalytic subset $A$ (see Definition 2.1.1 for a subanalytic set), if $A$ is 1-regular then, using the Curve Selection Lemma (see [5]), one proves that $\bar{A}$ is also 1-regular. Clearly, the converse does not hold. For example, let $X=\mathbb{R}^{2}$ with coordinates $(x, y), A=X \backslash\{x=0\}$ is not 1-regular at any point in the set $\{x=0\}$, but $\bar{A}=\mathbb{R}^{2}$ is 1 -regular at every point. Moreover a 1-regular set is locally connected at every point in $X$, that is, for any $p \in X$, there exists a family $\left\{V_{i}\right\}$ of fundamental neighborhoods of $p$ satisfying $V_{i} \cap A$ is connected.

Let $\left(x_{1}, x_{2}, \ldots, x_{n}\right)$ be a system of coordinates of $\mathbb{R}^{n}$ and $V \subset \mathbb{R}^{n}$ a 1regular relatvely compact open subset. Let us recall that, given $s>1$ and $h>0$, the space $\mathscr{C}^{s, h}(\bar{V})$ consists of the set of $f \in \mathscr{C}^{\infty}(V)$ whose arbitrary partial derivative extends to a continuous function on $\bar{V}$ with the following growth condition. There exists $C>0$ such that, for any $\alpha \in\left(\mathbb{Z}_{\geq 0}\right)^{n}$,

$$
\sup _{x \in \bar{V}}\left|D^{\alpha} f(x)\right| \leq C h^{|\alpha|}(|\alpha| !)^{s}
$$

where $D^{\alpha}:=\left(\frac{\partial}{\partial x_{1}}\right)^{\alpha_{1}} \ldots\left(\frac{\partial}{\partial x_{n}}\right)^{\alpha_{n}}$ for $\alpha=\left(\alpha_{1}, \ldots, \alpha_{n}\right) \in\left(\mathbb{Z}_{\geq 0}\right)^{n}$.

We denote by $\mathscr{D}_{\bar{V}}^{s, h}$ the set of functions $f \in \mathscr{C}^{s, h}(\bar{V})$ with $\left.D^{\alpha} f\right|_{\bar{V} \backslash V}=0$ for any $\alpha \in\left(\mathbb{Z}_{\geq 0}\right)^{n}$. The spaces $\mathscr{C}^{s, h}(\bar{V})$ and $\mathscr{D}_{\bar{V}}^{s, h}$ endowed with the norm

$$
\|f\|_{\bar{V}, s, h}:=\sup _{\substack{x \in \bar{V} \\ \alpha \in\left(\mathbb{Z}_{\geq 0}\right)^{n}}} \frac{\left|D^{\alpha} f(x)\right|}{h^{|\alpha|}(|\alpha| !)^{s}}
$$

are Banach spaces.

Given an open set $U \subset \mathbb{R}^{n}$, we set

$$
\begin{aligned}
& \mathscr{C}^{(s)}(U):=\lim _{V \Subset U} \lim _{h>0} \mathscr{C}^{s, h}(\bar{V}),
\end{aligned}
$$

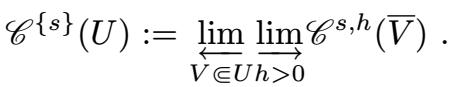

Here $V$ runs through the family of 1-regular relatively compact open subsets of $U$.

In [9] (see also [16]), it is proved that, given open sets $W, W^{\prime} \subset \mathbb{R}^{n}$ and a real analytic isomorphism $\Phi: W \rightarrow W^{\prime}$, the morphism $\cdot \circ \Phi: \mathscr{C}^{*}\left(W^{\prime}\right) \rightarrow \mathscr{C}^{*}(W)$ is an isomorphism. Hence, for an open subset $U$ in a real analytic mamifold $X$, the set of ultra-differentiable functions $\mathscr{C}^{*}(U)$ is also well-defined.

Let $X$ be a real analytic manifold. One checks easily that $\mathscr{C}^{\{s\}}$ and $\mathscr{C}^{(s)}$ are sheaves on $X$.

Definition 1.1.2. - For a locally closed subset $A$ in $X$, the image sheaf $j_{A}\left(\mathscr{C}^{*}\right) \subset \mathcal{J}_{A}$ is called the sheaf of Whitney jets of class * over $A$, and we denote it by $W_{A}^{*}$. 
Note that $W_{A}^{*}$ is a sheaf of rings and a $\mathscr{D}_{X}$-module. By the existence of partitions of the unity, the definition of $W_{A}^{*}$ is equivalent to the following. Given $F \in \mathcal{J}_{A}(U)$, then $F$ belongs to $W_{A}^{*}(U)$ if and only if there exists $f \in \mathscr{C}^{*}(U)$ with $j_{A}(f)=F$.

It follows from the definition that for any $F \in W_{A}^{*}(U)$ there exists an unique $\tilde{F} \in W_{\bar{A}}^{*}(U)$ such that $j_{A, \bar{A}}(\tilde{F})=F$. Hence the restriction map

$$
j_{A, \bar{A}}: W_{A}^{*}(U) \rightarrow W_{A}^{*}(U)
$$

is an isomorphism.

Let $A \subset \mathbb{R}^{n}$ be a locally closed set, $U \subset \mathbb{R}^{n}$ an open set and $s>1$. We introduce two families of semi-norms on $\mathscr{J}_{A}(U)$.

For $h>0$ and $K \subset A \cap U$, set

$$
\|F\|_{K, s, h}:=\sup _{\substack{y \in K \\ \alpha \in\left(\mathbb{Z}_{\geq 0}\right)^{n}}} \frac{\left|f_{\alpha}(y)\right|}{|\alpha| !^{s} h^{|\alpha|}} \quad\left(F=\left\{f_{\alpha}\right\}_{\alpha \in\left(\mathbb{Z}_{\geq 0}\right)^{n}} \in \mathcal{J}_{A}(U)\right) .
$$

We define, for $h>0$ and $K \subset A \cap U$, another semi-norm $\|\cdot\|_{K, s, h}^{W}$ on $\mathcal{J}_{A}(U)$ in the following manner. Given $F=\left\{f_{\alpha}\right\}_{\alpha \in\left(\mathbb{Z}_{\geq} 0\right)^{n}} \in \mathcal{J}_{A}(U)$ and $\beta \in\left(\mathbb{Z}_{\geq 0}\right)^{n}$ and $x, x^{\prime} \in A \cap U$, we set

$$
\begin{aligned}
S_{\beta} F & :=\left\{f_{\alpha+\beta}\right\}_{\alpha}, \\
T_{m}\left(F ; x, x^{\prime}\right) & :=\sum_{|\alpha| \leq m} \frac{1}{\alpha !} f_{\alpha}\left(x^{\prime}\right)\left(x-x^{\prime}\right)^{\alpha}, \\
R_{m}\left(F ; x, x^{\prime}\right) & :=f_{0}(x)-T_{m}\left(F ; x, x^{\prime}\right) .
\end{aligned}
$$

Then $\|F\|_{K, s, h}^{W}$ is defined by

$$
\sup _{m \geq 0, \alpha} \frac{m !}{(|\alpha|+m+1) !^{s} h^{|\alpha|+m+1}}\left(\sup _{x, x^{\prime} \in K, x \neq x^{\prime}} \frac{\left|R_{m}\left(S_{\alpha} F ; x, x^{\prime}\right)\right|}{\left|x-x^{\prime}\right|^{m+1}}\right) .
$$

The following characterization of $W_{A}^{*}$ can be found in [3].

THEOREM 1.1.3. - Let $A \subset \mathbb{R}^{n}$ be a locally closed set and $U \subset \mathbb{R}^{n}$ an open set.

1. A jet $F \in \mathcal{J}_{A}(U)$ belongs to $W_{A}^{(s)}(U)$ if and only if, for any $h>0$ and any compact set $K$ in $U,\|F\|_{A \cap K, s, h}<\infty$ and $\|F\|_{A \cap K, s, h}^{W}<\infty$ hold.

2. A jet $F \in \mathcal{J}_{A}(U)$ belongs to $W_{A}^{\{s\}}(U)$ if and only if, for any compact set $K$ in $U$, there exists $h>0$ such that $\|F\|_{A \cap K, s, h}<\infty$ and $\|F\|_{A \cap K, s, h}^{W}<\infty$. 
Let $A \subset X=\mathbb{R}^{n}$ be a compact set. We set

$$
W_{A}^{s, h}(X)=\left\{F \in \mathcal{J}_{A}(X) ;\|F\|_{A, s, h}+\|F\|_{A, s, h}^{W}<\infty\right\} .
$$

Endowing $W_{A}^{s, h}(X)$ with the norm $\|\cdot\|_{A, s, h}+\|\cdot\|_{A, s, h}^{W}$, it becomes a Banach space. It follows from Theorem 1.1.3 that

$$
\begin{aligned}
& W_{A}^{(s)}(X)=\lim _{h>0} W_{A}^{s, h}(X) \quad \text { and } \\
& W_{A}^{\{s\}}(X)={\underset{h>0}{\longrightarrow} W_{A}^{s, h}(X) .}^{\lim _{h>0}}
\end{aligned}
$$

It follows that $W_{A}^{*}(X)$ can be endowed with a locally convex topology by these projective or inductive limits. It is easy to see $W_{A}^{*}(X)$ is an FS space (resp. a DFS space) if $*=(s)$ (resp. $*=\{s\}$ ) respectively.

1.2. Ultradistributions. - For a complete presentation of the theory of ultradistributions, we cite [8].

Let $X$ be a real analytic manifold. Let us recall that, given a sheaf $F$ on $X$ and $U \subset X$ an open set, we denote by $\Gamma_{c}(U, F)$, the set of sections of $F$ on $U$ with compact support.

Given $U \subset \mathbb{R}^{n}$ open, the locally convex topological vector spaces $\mathscr{D}^{*}(U)$ and $\mathscr{D}^{s, h}(U)$ are defined as

$$
\begin{aligned}
& \mathscr{D}^{s, h}(U):={\underset{V \Subset U}{\vec{V}}}_{\lim _{\bar{V}}^{s, h}} \simeq \Gamma_{c}\left(U, \mathscr{C}^{s, h}\right), \\
& \mathscr{D}^{(s)}(U):=\lim _{V \Subset U} \varliminf_{h>0} \mathscr{D}_{\bar{V}}^{s, h} \simeq \Gamma_{c}\left(U, \mathscr{C}^{(s)}\right), \\
& \mathscr{D}^{\{s\}}(U):=\underset{V \Subset U}{\lim _{h>0}} \underset{\lim _{h}}{\longrightarrow} \mathscr{D}_{\bar{V}}^{s, h} \simeq \Gamma_{c}\left(U, \mathscr{C}^{\{s\}}\right),
\end{aligned}
$$

where $V$ runs through the family of 1-regular relatively compact open subsets of $U$.

In [9] (see also [16]), it is proved that, given open sets $W, W^{\prime} \subset \mathbb{R}^{n}$ and a real analytic isomorphism $\Phi: W \rightarrow W^{\prime}$, the morphism $\circ \Phi: \mathscr{D}^{*}\left(W^{\prime}\right) \rightarrow \mathscr{D}^{*}(W)$ is an isomorphism. Hence we can define $\mathscr{D}^{*}(U)$ for an open set $U$ in a real analytic manifold.

DEFINITION 1.2.1. - $\quad$ 1. Let $X$ be a real analytic manifold of dimension $n$. We denote by $\mathscr{V}^{*}$ the sheaf on $X$ of volume elements with coefficients in $\mathscr{C}^{*}$, that is $\mathscr{V}^{*}:=\mathscr{C}^{*} \underset{\mathscr{G}}{\otimes} \omega_{X}^{(n)} \underset{\mathbb{Z}}{\otimes}$ or ${ }_{X}$, where $\omega_{X}^{(n)}$ (resp. $\left.\mathscr{C}\right)$ is the sheaf of real analytic $n$-forms (resp. functions) on $X$, and $o r_{X}$ is that of orientations on $X$. 
2. Let $U$ be an open subset of $X$. The space of ultradistributions on $U$ of class $(s)$ of Beurling type (resp. of class $\{s\}$ of Roumieu type), or simply of class $(s)$ (resp. $\{s\})$, denoted $\mathscr{D} b^{(s)}(U)$ (resp. $\left.D b^{\{s\}}(U)\right)$, is defined as the strong dual space of $\Gamma_{c}\left(U, \mathscr{V}^{(s)}\right)$ (resp. $\left.\Gamma_{c}\left(U, \mathscr{V}^{\{s\}}\right)\right)$.

In [17], it is proved that $\mathscr{D} b^{*}$ is a sheaf on $X$.

Given a closed set $Z \subset X$ and $F \in \operatorname{Mod}\left(\mathbb{C}_{X}\right)$ denote by $\Gamma_{Z}(F)$ the subsheaf of $F$ of sections supported by $Z$.

If $A$ is a compact subset of $X=\mathbb{R}^{n}$, from results of H. Whitney and J.M. Kantor, it follows that the topological dual of $W_{A}^{*}(X)$ is isomorphic to $\Gamma_{A}\left(X, D b^{*}\right)$.

DEFINITION 1.2.2. - The presheaf of tempered ultradistributions of class $*$ on $X$, denoted $\mathscr{D} b_{X}^{* t}$, is defined by

$$
\mathscr{D} b_{X}^{* t}(U):=\frac{\Gamma\left(X ; \mathscr{D} b^{*}\right)}{\Gamma_{X \backslash U}\left(X ; \mathscr{D} b^{*}\right)}, \quad U \subset X \text { open } .
$$

Note that $\mathscr{D} b_{X}^{* t}$ is not a sheaf on $X$.

In Proposition 1.2.3 below (originally due to $\mathrm{H}$. Komatsu), we recall a sufficient condition for a continuous function to extend to $\mathbb{R}^{n}$ as an ultradistribution. For $U$ an open subset of $\mathbb{R}^{n}$, we denote by $D b^{s, l}(U)$ the dual space of $\mathscr{D}^{s, l}(U)$, and by $L_{\mathrm{loc}}^{1}(U)$ the set of locally integrable functions on $U$.

Proposition 1.2.3. - Let $U \subset \mathbb{R}^{n}$ be an open set and $f \in L_{\text {loc }}^{1}(U)$. Suppose that there exist positive constants $h$ and $C$ satisfying

$$
|f(x)| \leq C \exp \left(\frac{h}{\operatorname{dist}(x, \partial U)^{\frac{1}{s-1}}}\right) .
$$

Then we have $f \in \mathscr{D} b_{X}^{(s) t}(U)$.

We need some technical results.

LEMMA 1.2.4. - Let $U$ be a relatively compact open subset of $\mathbb{R}^{n}$.

1. Let $f \in L_{\mathrm{loc}}^{1}(U)$. If there exist positive constants $l$ and $M$ such that, for any $\varphi \in \mathscr{D}^{s, l}(U)$,

$$
\left|\int_{U} f \varphi d x\right| \leq M\|\varphi\|_{U, s, l}
$$

then $f$ extends to an ultradistribution in $\mathscr{D} b^{s, l}\left(\mathbb{R}^{n}\right)$. In particular, $f$ belongs to $\mathscr{D} b_{X}^{(s) t}(U)$. 
2. There exist constants $C>0$ and $\kappa>0$ such that, for any $l>0$ and for any $\varphi \in \mathscr{D}^{s, l}(U)$, the inequality

$$
|\varphi(x)| \leq C \exp \left(\frac{-\kappa l^{\frac{-1}{s-1}}}{\operatorname{dist}(x, \partial U)^{\frac{1}{s-1}}}\right)\|\varphi\|_{U, s, l}
$$

holds for any $x \in U$.

Proof. - (i). By the Hahn-Banach's extension Theorem the functional $\int_{U} f$. $d x$ extends to $\mathscr{D}^{s, l}\left(\mathbb{R}^{n}\right)$.

(ii). Set

$$
M:=\sup _{\substack{\alpha \in\left(\mathbb{Z}_{\geq 0}\right)^{n} \\ x \in U}} \frac{\left|D^{\alpha} \varphi(x)\right|}{(|\alpha| !)^{s} l^{|\alpha|}},
$$

and let $y$ be a point in $\partial U$ with $|x-y|=\operatorname{dist}(x, \partial U)$. Then we have

$$
\varphi(x)=\frac{1}{(k-1) !} \int_{0}^{1}(1-t)^{k-1} \frac{d^{k}}{d t^{k}} \varphi((x-y) t+y) d t \quad \text { for any } k \in \mathbb{N} .
$$

As

$$
\begin{aligned}
\frac{d^{k}}{d t^{k}} \varphi((x-y) t+y) & =\left.\left(\sum_{i=1}^{n}\left(x_{i}-y_{i}\right) \frac{\partial}{\partial z_{i}}\right)^{k} \varphi(z)\right|_{z=(x-y) t+y} \\
& =\left.k ! \sum_{|\alpha|=k} \frac{(x-y)^{\alpha}}{\alpha !} D_{z}^{\alpha} \varphi(z)\right|_{z=(x-y) t+y}
\end{aligned}
$$

hold, we obtain

$$
|\varphi(x)| \leq k(l|x-y|)^{k}(k !)^{s} M \sum_{|\alpha|=k} \frac{1}{\alpha !} \leq(2 n l|x-y|)^{k}(k !)^{s-1} M .
$$

Hence we have

$$
|\varphi(x)| \leq \inf _{k \geq 1}(2 n l|x-y|)^{k}(k !)^{s-1} M=\left(\inf _{k \geq 1} \epsilon^{k} k !\right)^{s-1} M
$$

where we set $\epsilon:=(2 n l|x-y|)^{\frac{1}{s-1}}=\left(2 n l \operatorname{dist}(x, \partial U)^{\frac{1}{s-1}}\right.$. We may assume $\epsilon \leq 1$. Then, for an integer $j_{0} \geq 1$ satisfying $j_{0} \leq \frac{1}{\epsilon}<j_{0}+1$, we get

$$
\begin{aligned}
\inf _{k \geq 1} \epsilon^{k} k ! & \leq \epsilon^{j_{0}} j_{0} ! \leq\left(\frac{1}{j_{0}}\right)^{j_{0}} j_{0} ! \leq e^{\frac{1}{12}} \sqrt{j_{0}} e^{-j_{0}} \\
& \leq e^{\frac{13}{12}} \frac{1}{\sqrt{\epsilon}} \exp \left(-\frac{1}{\epsilon}\right) \leq 2 e^{\frac{13}{12}} \exp \left(-\frac{1}{2 \epsilon}\right)
\end{aligned}
$$

thanks to the Stirling formula

$$
j !=\sqrt{2 \pi} j^{j+\frac{1}{2}} e^{-j+\frac{\vartheta(j)}{12 j}} \quad 0<\vartheta(j)<1 .
$$

This completes the proof. 
Proof of Proposition 1.2.3.. - Since the problem is local, we may assume that $U$ is relatively compact. Lemma 1.2.4 (ii) implies that there exists $C^{\prime}>0$ and $\kappa>0$ such that, for any $l>0$ and for any $\varphi \in \mathscr{D}^{s, l}(U)$,

$$
|f(x) \varphi(x)| \leq C^{\prime} \exp \left(\frac{h-\kappa l^{\frac{-1}{s-1}}}{\operatorname{dist}(x, \partial U)^{\frac{1}{s-1}}}\right)\|\varphi\|_{\bar{U}, s, l} .
$$

If we take $l>0$ sufficiently small, then the conclusion follows from Lemma 1.2.4 (i).

1.3. An example. - Let $l \geq 1$. Set

$$
\begin{gathered}
U_{1}:=\left\{(x, y) \in \mathbb{R}^{2} ; y>x^{2 l+1}\right\}, \\
U_{2}:=\mathbb{R} \times \mathbb{R}_{<0} .
\end{gathered}
$$

Define a function $u_{i}(x, y) \in L_{\text {loc }}^{1}\left(U_{i}\right)(i=1,2)$ by

$$
\begin{aligned}
& u_{1}(x, y):= \begin{cases}\exp \left(\frac{1}{y-x^{2 l+1}}\right) & \text { for }(x, y) \in U_{1} \cap\left(\mathbb{R} \times \mathbb{R}_{>0}\right) \\
0 & \text { for }(x, y) \in U_{1} \cap\left(\mathbb{R} \times \mathbb{R}_{\leq 0}\right)\end{cases} \\
& u_{2}(x, y):=0 \text { for any }(x, y) \in U_{2}
\end{aligned}
$$

By Proposition 1.2.3, $u_{1} \in \mathscr{D} b^{(2) t}\left(U_{1}\right)$. Clearly $u_{2} \in \mathscr{D} b^{(2) t}\left(U_{2}\right)$. As $\left.u_{1}\right|_{U_{1} \cap U_{2}}=\left.u_{2}\right|_{U_{1} \cap U_{2}}$, there exists $u \in L_{\text {loc }}^{1}\left(U_{1} \cup U_{2}\right)$ such that $\left.u\right|_{U_{1}}=u_{1}$ and $\left.u\right|_{U_{2}}=u_{2}$, but $u \notin \mathscr{D} b^{(2) t}\left(U_{1} \cup U_{2}\right)$. Indeed, let us prove that $u \notin \mathscr{D} b^{(2) t}\left(U_{1} \cup U_{2}\right)$.

Set $U:=U_{1} \cup U_{2}$ and $D:=\left\{(x, y) \in \mathbb{R}^{2} ;|x|<1,|y|<1\right\}$. Suppose that $u \in \mathscr{D} b^{(2) t}(U)$. Then there exist positive constants $C$ and $h$ such that

$$
\left|\int_{U} u \psi d x\right| \leq C\|\psi\|_{\bar{D}, 2, h}
$$

holds for any $\psi \in \mathscr{D}^{(2)}(U \cap D)$. Now applying Lemma A.6 to the situation $\varphi(t)=t^{2}, s=2$ and $K=\{0\} \subset \mathbb{R}^{2}$, we obtain a family of functions $\left\{\chi_{\epsilon}(x, y)\right\}_{\epsilon>0}$ satisfying the conditions 1., 2. and 3. of the lemma. Set

$$
D_{\epsilon}:=\left\{-\frac{5 \epsilon}{2} \leq x \leq-\frac{3 \epsilon}{2}, 0 \leq y \leq x^{2 l+1}\right\}
$$

and

$$
\psi_{\epsilon}(x, y):=\frac{\chi_{\epsilon}(x+2 \epsilon, y)}{C_{h} \exp \left(\varphi\left(\epsilon^{-1}\right)\right)}=\frac{\exp \left(-\epsilon^{-2}\right) \chi_{\epsilon}(x+2 \epsilon, y)}{C_{h}}
$$

BULletin DE LA SOCiÉtÉ MATHÉmAtiqUe DE FRANCE 
where $C_{h}>0$ is the positive constant given in Lemma A.6 1. Remark that $\left\|\psi_{\epsilon}\right\|_{\bar{D}, 2, h} \leq 1$ holds for any $\epsilon>0$, and hence, $\left|\int_{U} u \psi_{\epsilon} d x\right|$ is uniformly bounded. On the other hand, for sufficiently small $\epsilon>0$, we have

$$
\left.\psi_{\epsilon}\right|_{D_{\epsilon}}=\frac{1}{C_{h}} \exp \left(-\epsilon^{-2}\right)
$$

and

$$
\exp \left(\frac{1}{y-x^{2 l+1}}\right) \geq \exp \left(\frac{1}{-2 x^{2 l+1}}\right) \geq \exp \left(\kappa \epsilon^{-(2 l+1)}\right) \quad\left((x, y) \in D_{\epsilon}\right)
$$

for some positive constant $\kappa>0$. Therefore we have

$$
\int_{U} u \psi_{\epsilon} d x \geq \int_{D_{\epsilon}} u \psi_{\epsilon} d x \geq \frac{1}{C_{h}} \exp \left(-\epsilon^{-2}+\kappa \epsilon^{-(2 l+1)}\right) \int_{D_{\epsilon}} d x \rightarrow \infty
$$

which give a contradiction. Hence, we conclude that $u \notin \mathscr{D} b^{(2) t}\left(U_{1} \cup U_{2}\right)$.

It follows that tempered distributions do no glue on finite coverings of open sets with smooth boundaries. In particular, the presheaf $\mathscr{D} b_{X}^{* t}$ is not a sheaf on the subanalytic site relative to $\mathbb{R}^{2}$ (see Subsection 2.1 for the definition of subanalytic site).

Among the purpose of this paper there is the attempt to overcome the difficulty presented in this example. We will define a sheaf on the subanalytic site of stratified Whitney jets of Gevrey order. Then, we will define stratified ultradistributions. In the end, by means of stratified ultradistributions, we will define tempered-stratified ultradistributions and we will prove two results. The first states that, if $X$ is a real surface, tempered-stratified ultradistributions define a sheaf on the subanalytic site relative to $X$. The second states that the sections of tempered-stratified ultradistributions on open subanalytic open sets with 1-regular complementary coincides with sections of tempered ultradistributions.

\section{Stratified Whitney jets and stratified ultradistributions}

2.1. Review on the subanalytic site. - Let $X$ be a real analytic manifold countable at infinity.

Definition 2.1.1. - $\quad$ 1. A set $Z \subset X$ is said semi-analytic at $x \in X$ if the following condition is satisfied. There exists an open neighborhood $W$ of $x$ such that $Z \cap W=\cup_{i \in I} \cap_{j \in J} Z_{i j}$ where $I$ and $J$ are finite sets and either $Z_{i j}=\left\{y \in X ; f_{i j}(y)>0\right\}$ or $Z_{i j}=\left\{y \in X ; f_{i j}(y)=0\right\}$ for some real-valued real analytic functions $f_{i j}$ on $W$. Further, $Z$ is said semi-analytic if $Z$ is semi-analytic at any $x \in X$.

TOME $139-2011-\mathrm{N}^{\mathrm{O}} 3$ 
2. A set $Z \subset X$ is said subanalytic if the following condition is satisfied. For any $x \in X$, there exist an open neighborhood $W$ of $x$, a real analytic manifold $Y$ and a relatively compact semi-analytic set $A \subset X \times Y$ such that $\pi(A)=Z \cap W$, where $\pi: X \times Y \rightarrow X$ is the projection.

Given $Z \subset X$, denote by $\check{Z}$ (resp. $\bar{Z}, \partial Z$ ) the interior (resp. the closure, the boundary) of $Z$.

Proposition 2.1.2 (See [1]). - Let $Z$ and $V$ be subanalytic subset of $X$. Then $Z \cup V, Z \cap V, \bar{Z}, \dot{Z}$ and $Z \backslash V$ are subanalytic. Moreover the connected components of $Z$ are subanalytic, the family of connected components of $Z$ is locally finite and $Z$ is locally connected at any point in $Z$.

Definition 2.1.3. - 1. A family $\left\{A_{\alpha}\right\}_{\alpha \in \Lambda}$ of subanalytic subsets of $X$ is said a stratification of $X$ if $\left\{A_{\alpha}\right\}_{\alpha \in \Lambda}$ is locally finite, $X=\bigsqcup_{\alpha \in \Lambda} A_{\alpha}$ and each $A_{\alpha}$ is a locally closed subanalytic manifold.

2. Given a locally closed subanalytic set $A \subset X$, we say that $\left\{A_{\alpha}\right\}_{\alpha \in \Lambda}$ is a stratification of $A$ if $A$ is the disjoint union of the $A_{\alpha}$ and there exists a stratification $\left\{A_{\alpha}\right\}_{\alpha \in \Lambda \cup \Lambda^{\prime}}$ of $X$ finer than the stratification $\{A, X \backslash A\}$.

3. Let $A$ be a subanalytic subset of $X,\left\{A_{\alpha}\right\}_{\alpha \in \Lambda}$ a stratification of $A$. Then $\left\{A_{\alpha}\right\}_{\alpha \in \Lambda}$ is called a 1-regular strafication if each stratum is 1-regular, connected and relatively compact.

finite family of subsets of

Proposition 2.1.4 (See [10]). - $\quad$ 1. Let $Z \subset X$ be a locally closed subanalytic subset. There exists a 1-regular stratification of $Z$.

2. Let $U \subset X$ be a subanalytic open set. There exists an open covering $\left\{U_{j}\right\}_{j \in J}$ of $U$ such that, for any $j \in J, U_{j}$ is a subanalytic 1-regular set and for any compact $K \subset X$ there exists a finite set $J_{K} \subset J$ such that $K \cap\left(\cup_{j \in J_{K}} U_{j}\right)=K \cap U$.

For the rest of the subsection we refer to [7].

We denote by $\operatorname{Op}(X)$ the family of open subsets of $X$. For $k$ a commutative ring, we denote by $\operatorname{Mod}\left(k_{X}\right)$ the category of sheaves of $k$-modules on $X$.

Let us recall the definition of the subanalytic site $X_{\text {sa }}$ relative to $X$. An element $U \in \mathrm{Op}(X)$ is an open set for $X_{\mathrm{sa}}$ if it is open, relatively compact and subanalytic. The family of open sets of $X_{\mathrm{sa}}$ is denoted $\mathrm{Op}^{c}\left(X_{\mathrm{sa}}\right)$. For $U \in \operatorname{Op}^{c}\left(X_{\mathrm{sa}}\right)$, a subset $S$ of the family of open subsets of $U$ is said an open covering of $U$ in $X_{\mathrm{sa}}$ if $S \subset \mathrm{Op}^{c}\left(X_{\mathrm{sa}}\right)$ and there exists a finite subset $S_{0} \subset S$ such that $\cup_{V \in S_{0}} V=U$. The set of coverings of $U$ in $X_{\mathrm{sa}}$ is denoted by $\operatorname{Cov}_{\mathrm{sa}}(U)$.

We denote by $\operatorname{Mod}\left(k_{X_{\mathrm{sa}}}\right)$ the category of sheaves of $k$-modules on the subanalytic site. With the aim of defining the category $\operatorname{Mod}\left(k_{X_{\mathrm{sa}}}\right)$, the adjective 
"relatively compact" can be omitted in the definition above. Indeed, in [7, Remark 6.3.6], it is proved that $\operatorname{Mod}\left(k_{X_{\mathrm{sa}}}\right)$ is equivalent to the category of sheaves on the site whose open sets are the open subanalytic subsets of $X$ and whose coverings are locally finite and consist of open subanalytic sets.

Let $\operatorname{PSh}\left(k_{X_{\mathrm{sa}}}\right)$ be the category of presheaves of $k$-modules on $X_{\mathrm{sa}}$. Denote by for $: \operatorname{Mod}\left(k_{X_{\mathrm{sa}}}\right) \rightarrow \operatorname{PSh}\left(k_{X_{\mathrm{sa}}}\right)$ the forgetful functor which associates to a sheaf $F$ on $X_{\text {sa }}$ its underlying presheaf. It is well known that for admits a left adjoint ${ }^{a}: \operatorname{PSh}\left(k_{X_{\mathrm{sa}}}\right) \rightarrow \operatorname{Mod}\left(k_{X_{\mathrm{sa}}}\right)$.

For $F \in \operatorname{PSh}\left(k_{X_{\mathrm{sa}}}\right)$, let us briefly recall the construction of $F^{a}$.

For $U \in \operatorname{Op}^{c}\left(X_{\mathrm{sa}}\right)$ and $S \in \operatorname{Cov}_{\mathrm{sa}}(U)$, let $F(S)$ be defined as the kernel of the morphism

$$
\begin{aligned}
& \prod_{U \in S} F(U) \longrightarrow \prod_{U, V \in S} F(U \cap V) \\
& \left\{s_{U}\right\}_{U \in S} \longmapsto\left\{\left.s_{U}\right|_{U \cap V}-\left.s_{V}\right|_{U \cap V}\right\}_{U, V \in S} .
\end{aligned}
$$

If $S^{\prime} \in \operatorname{Cov}_{\mathrm{sa}}(U)$ is a refinement of $S$, then there exists a natural morphism $F(S) \longrightarrow F\left(S^{\prime}\right)$.

Now, for $U \in \mathrm{Op}^{c}\left(X_{\mathrm{sa}}\right)$, set

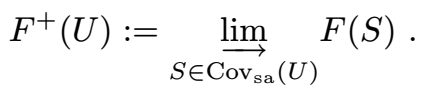

It turns out that $F^{a} \simeq F^{++}$.

The following Lemma is an immediate consequence of the defintions above.

Lemma 2.1.5. - Let $\mathcal{T} \subset \mathrm{Op}\left(X_{\mathrm{sa}}\right)$ be such that for any $U \in \mathrm{Op}\left(X_{\mathrm{sa}}\right)$ there exists $S \in \operatorname{Cov}_{\mathrm{sa}}(U)$, such that $S \subset \mathcal{J}$. Let $F, G \in \operatorname{PSh}\left(k_{X_{\mathrm{sa}}}\right)$ and suppose that there exists a morphism of presheaves $\varphi: F \rightarrow G$ such that, for any $V \in \mathcal{J}$, $\varphi_{V}: F(V) \stackrel{\sim}{\rightarrow} G(V)$. Then $F^{a} \simeq G^{a}$.

We denote by

$$
\varrho: X \longrightarrow X_{\mathrm{sa}},
$$

the natural morphism of sites associated to $\mathrm{Op}^{c}\left(X_{\mathrm{sa}}\right) \longrightarrow \mathrm{Op}(X)$. We refer to [7] for the definitions of the functors $\varrho_{*}: \operatorname{Mod}\left(k_{X}\right) \longrightarrow \operatorname{Mod}\left(k_{X_{\mathrm{sa}}}\right)$ and $\varrho^{-1}: \operatorname{Mod}\left(k_{X_{\mathrm{sa}}}\right) \longrightarrow \operatorname{Mod}\left(k_{X}\right)$ and for Proposition 2.1.6 below.

Proposition 2.1.6. - $\quad$ 1. The functor $\varrho^{-1}$ is left adjoint to $\varrho_{*}$.

2. The functor $\varrho^{-1}$ has a left adjoint denoted by $\varrho_{!}: \operatorname{Mod}\left(k_{X}\right) \rightarrow \operatorname{Mod}\left(k_{X_{\mathrm{sa}}}\right)$.

3. The functors $\varrho^{-1}$ and $\varrho$ ! are exact, $\varrho_{*}$ is exact on constructible sheaves.

4. The functors $\varrho_{*}$ and $\varrho_{\text {! }}$ are fully faithful.

Through $\varrho_{*}$, we will consider $\operatorname{Mod}\left(k_{X}\right)$ as a subcategory of $\operatorname{Mod}\left(k_{X_{\mathrm{sa}}}\right)$.

The functor $\varrho$ ! is described as follows. If $U \in \mathrm{Op}^{c}\left(X_{\mathrm{sa}}\right)$ and $F \in \operatorname{Mod}\left(k_{X}\right)$, then $\varrho_{!}(F)$ is the sheaf on $X_{\mathrm{sa}}$ associated to the presheaf $U \mapsto F(\bar{U})$. 
2.2. Stratified Whitney jets. - Let $A$ be a locally closed subanalytic subset in a real analytic manifold $X$ and $U \subset X$ an open subset. Let us define the sheaf $\delta W_{A}^{*}$ of stratified Whitney jets of class $*$ over $A$.

Definition 2.2.1. - $\quad$ 1. We say that $F \in \mathcal{J}_{A}(U)$ is a stratified Whitney jet of class * over $A$ in $U$ if for any compact subanalytic set $K$ in $U$ there exists a subanalytic stratification $\left\{A_{\alpha}\right\}_{\alpha \in \Lambda}$ of $A$ such that $j_{A_{\alpha} \cap K, A}(F) \in$ $W_{A_{\alpha} \cap K}^{*}(U)$ holds for any $\alpha \in \Lambda$.

2. We denote by $\& W_{A}^{*}(U)$ the set of stratified Whitney jets of class $*$ over $A$ in $U$.

It is easy to verify that $\phi W_{A}^{*}$ is a sheaf on $X$.

EXAmple 2.2.2. - Later we will prove that, if the set $A$ is 1-regular, then $W_{A}^{*}=\phi W_{A}^{*}$. However, in general, $W_{A}^{*}$ and $\delta W_{A}^{*}$ are different. For example, let $m \geq 2, X=\mathbb{R}^{2}$ with coordinates $(x, y)$ and

$$
B=\left\{(x, y) \in \mathbb{R}^{2} ; y=0, x \geq 0\right\}, \quad B_{m}=\left\{(x, y) \in \mathbb{R}^{2} ; y=x^{m}, x \geq 0\right\} .
$$

Set $A=B \cup B_{m}$. We define the jet $F=\left\{f_{\alpha}\right\} \in \mathcal{J}_{A}(X)$ by:

$$
f_{\alpha}(x, y)=\left\{\begin{array}{ll}
0 & (x, y) \in B_{m} \\
\frac{\partial^{\alpha}}{\partial x^{\alpha}} \exp \left(-\frac{1}{x}\right) & (x, y) \in B
\end{array} .\right.
$$

Then $F \in \phi W_{A}^{\{2\}}(X)$, but $F \notin W_{A}^{\{2\}}(X)$. As a matter of fact, if $F \in W_{A}^{\{2\}}(X)$, then we can find $\tilde{F}=\left\{\tilde{f}_{\alpha}\right\}_{\alpha} \in \phi \mathcal{W}_{D}^{\{2\}}(X)$ with $j_{\partial D, D}(\tilde{F})=F$ where

$$
D=\left\{(x, y) \in \mathbb{R}^{2} ; 0 \leq x, 0 \leq y \leq x^{m}\right\} .
$$

By applying Lemma A.3 to $\tilde{F}$ and $D$ with the 1-regular stratification $\{D \backslash$ $\left.\partial D, B \backslash\{0\}, B_{m} \backslash\{0\},\{0\}\right\}$, we have constants $C, l>0$ satisfying

$$
\left|f_{0}(x, 0)\right|=\left|\tilde{f}_{0}(x, 0)\right| \leq C \exp \left(-\frac{l}{x^{m}}\right) \quad(x>0),
$$

which is impossible.

We can also give the similar example on an open subanalytic set. Set $U:=$ $X \backslash D$ (D was given by (2.2)), and define the jet $G=\left\{g_{\alpha}\right\} \in \mathcal{J}_{U}(X)$ by

$$
g_{\alpha}(x, y)=\left\{\begin{array}{ll}
0 & (x, y) \in U \backslash\{x>0, y<0\} \\
\frac{\partial^{\alpha}}{\partial x^{\alpha}} \exp \left(-\frac{1}{x}\right) & (x, y) \in\{x>0, y<0\}
\end{array} .\right.
$$

Then $G \in \phi W_{U}^{\{2\}}(X)$, but $G \notin W_{U}^{\{2\}}(X)$. The reason is the same as that for the first example. 
REMARK 2.2.3. - 1. We cannot expect $\phi W_{A}^{*}(X)=\phi W_{A}^{*}(X)$ on the contrary to $W_{A}^{*}(X)=W_{A}^{*}(X)$. For example, consider the case $X=\mathbb{R}^{2}$ with coordinates $(x, y)$ and $A=X \backslash\{x=0\}$.

2. We have the equivalence, for every $F \in \mathcal{J}_{A}(U)$,

$$
j_{A_{\alpha} \cap K, A}(F) \in W_{A_{\alpha} \cap K}^{*}(U) \Longleftrightarrow j_{\cos _{A}\left(A_{\alpha} \cap K\right), A}(F) \in W_{\operatorname{clos}_{A}\left(A_{\alpha} \cap K\right)}^{*}(U)
$$

where $\operatorname{clos}_{A}(B)$ denotes the closure of the set $B$ in $A$. Hence, in Definition 2.2.1, the condition

$$
\text { " } j_{A_{\alpha} \cap K, A}(F) \in W_{A_{\alpha} \cap K}^{*}(U) \text { for any } \alpha \in \Lambda \text { " }
$$

can be replaced with

$$
\text { " } j_{\operatorname{clos}_{A}\left(A_{\alpha} \cap K\right), A}(F) \in W_{\operatorname{clos}_{A}\left(A_{\alpha} \cap K\right)}^{*}(U) \text { for any } \alpha \in \Lambda " .
$$

In particular, if $A$ is a compact subanalytic subset in $X$, then $F \in$ $8 W_{A}^{*}(X)$ if and only if there exists a stratification $\left\{A_{\alpha}\right\}_{\alpha \in \Lambda}$ such that $j_{\bar{A}_{\alpha}, A}(F) \in W_{\bar{A}_{\alpha}}^{*}(X)$.

The sheaf $W_{A}^{*}$ is a subsheaf of $\phi W_{A}^{*}$, and $\phi W_{A}^{*}$ is a sheaf of rings and a $\mathscr{D}_{X}$-module. Further, $W_{A}^{*}$ and $\delta W_{A}^{*}$ are $\mathscr{C}^{*}$-modules and soft sheaves. If $\left\{A_{\alpha}\right\}_{\alpha \in \Lambda}$ is a stratification of $A$, then we denote by $\delta W_{\left\{A_{\alpha}\right\}}^{*}$ the subsheaf of $\varnothing W_{A}^{*}$ defined by:

$$
\delta W_{\left\{A_{\alpha}\right\}}^{*}(U)=\left\{F \in \mathcal{J}_{A}(U) ; j_{A_{\alpha}, A}(F) \in W_{A_{\alpha}}^{*}(U) \text { for any } \alpha \in \Lambda\right\} .
$$

Lemma 2.2.4. - Let $A$ be a locally closed subanalytic subset in $X$, and let $\left\{A_{i}\right\}_{i=1}^{l}$ be a finite family of locally closed subanalytic subset in $X$ with $A=$ $\cup A_{i}$. We assume that every $A_{i}$ is a closed subset in $A$, or that every $A_{i}$ is open in $A$. Then the sequence of sheaves

$$
0 \rightarrow \phi W_{A}^{*} \rightarrow \underset{1 \leq i \leq k}{\oplus} \phi W_{A_{i}}^{*} \rightarrow \underset{1 \leq i<j \leq k}{\oplus} \phi W_{A_{i} \cap A_{j}}^{*}
$$

is exact.

Proof. - The injectivity of the second morphism of (2.3) is clear.

Under the condition that every $A_{i}$ is closed (or open) in $A$, the sequence of sheaves of jets

$$
0 \rightarrow \mathcal{J}_{A} \rightarrow \underset{1 \leq i \leq k}{\bigoplus} \mathcal{J}_{A_{i}} \rightarrow \underset{1 \leq i<j \leq k}{\oplus} \mathcal{J}_{A_{i} \cap A_{j}}
$$

is exact. Let $U$ be an open subset and $K$ a compact subanalytic subset in $U$, and let $F_{i} \in \delta W_{A_{i}}^{*}(U)$ with $j_{A_{i} \cap A_{j}, A_{i}}\left(F_{i}\right)=j_{A_{i} \cap A_{j}, A_{j}}\left(F_{j}\right)$. Then by the above exact sequence we can find a jet $F \in \mathcal{J}_{A}(U)$ with $j_{A_{i}, A}(F)=F_{i}$. To conclude the proof let us show that $F \in \phi W_{A}^{*}(U)$.

As $F_{i} \in \phi W_{A A_{i}}^{*}(U)$ there exists a stratification $\left\{A_{\alpha}^{(i)}\right\}_{\alpha}$ of $A_{i}$ such that $j_{A_{\alpha}^{(i)} \cap K, A_{i}}\left(F_{i}\right) \in \mathcal{W}_{A_{\alpha}^{(i)} \cap K}^{*}(U)$. If we take a stratification $\left\{A_{\alpha}\right\}$ of $A$ finer than 
any partition $\left\{\left\{A_{\alpha}^{(i)}\right\}_{\alpha}, A \backslash A_{i}\right\}$ of $A$, then we have $j_{A_{\alpha} \cap K, A_{i}}\left(F_{i}\right) \in \mathcal{W}_{A_{\alpha} \cap K}^{*}(U)$ for any stratum $A_{\alpha}$ with $A_{\alpha} \subset A_{i}$. Hence, for each $A_{\alpha}$ we conclude

$$
j_{A_{\alpha} \cap K, A}(F)=j_{A_{\alpha} \cap K, A_{i}}\left(F_{i}\right) \in W_{A_{\alpha} \cap K}^{*}(U)
$$

where the index $i$ is taken so that $A_{\alpha} \subset A_{i}$.

Remark that, in general, the sequence

$$
0 \rightarrow W_{A}^{*} \rightarrow \underset{1 \leq i \leq k}{\oplus} W_{A_{i}}^{*} \rightarrow \underset{1 \leq i<j \leq k}{\oplus} W_{A_{i} \cap A_{j}}^{*}
$$

is not exact.

The following lemma is fundamental.

Lemma 2.2.5. - Let $X=\mathbb{R}^{n}$ and $A$ a locally closed subanalytic subset in $X$. For any $F=\left\{f_{\alpha}\right\} \in \& W_{A}^{*}(X)$ and any subanalytic curve $l \subset A$ joining $x, x^{\prime} \in A$, we have

$$
\left|R_{m}\left(F ; x, x^{\prime}\right)\right| \leq \frac{(\sqrt{n}|l|)^{m+1}}{m !} \sup _{\substack{|\alpha|=m+1 \\ y \in l}}\left|f_{\alpha}(y)\right|
$$

where $|l|$ denotes the length of the curve $l$.

Proof. - We recall the following formula of [18]. Let $l \subset A$ be a subanalytic curve, for any $x_{1}, x_{2}, x_{3} \in l$

$$
T_{m}\left(F ; x_{1}, x_{2}\right)-T_{m}\left(F ; x_{1}, x_{3}\right)=\sum_{|\beta| \leq m} \frac{R_{m-|\beta|}\left(S_{\beta} F ; x_{2}, x_{3}\right)}{\beta !}\left(x_{1}-x_{2}\right)^{\beta} .
$$

Noticing that

$$
R_{m-|\beta|}\left(S_{\beta} F ; x_{2}, x_{3}\right)=\sum_{|\gamma|=m-|\beta|+1} \frac{f_{\beta+\gamma}\left(x_{3}\right)}{\gamma !}\left(x_{2}-x_{3}\right)^{\gamma}+R_{m-|\beta|+1}\left(S_{\beta} F ; x_{2}, x_{3}\right),
$$

we have

$$
\begin{aligned}
\left|T_{m}\left(F ; x_{1}, x_{2}\right)-T_{m}\left(F ; x_{1}, x_{3}\right)\right| & \leq \sum_{|\beta| \leq m} \sum_{|\gamma|=m-|\beta|+1}\left|\frac{f_{\beta+\gamma}\left(x_{3}\right)}{\beta ! \gamma !}\left(x_{1}-x_{2}\right)^{\beta}\left(x_{2}-x_{3}\right)^{\gamma}\right| \\
& +\sum_{|\beta| \leq m}\left|\frac{R_{m-|\beta|+1}\left(S_{\beta} F ; x_{2}, x_{3}\right)}{\beta !}\left(x_{1}-x_{2}\right)^{\beta}\right| .
\end{aligned}
$$


The first term is estimated as follows. Suppose that $x_{1}, x_{2}, x_{3}$ are in a sequential order along $l$, then we have

$$
\begin{aligned}
& \sum_{|\beta| \leq m} \sum_{|\gamma|=m-|\beta|+1}\left|\frac{f_{\beta+\gamma}\left(x_{3}\right)}{\beta ! \gamma !}\left(x_{1}-x_{2}\right)^{\beta}\left(x_{2}-x_{3}\right)^{\gamma}\right| \\
& \leq\left(\sup _{y \in l,|\alpha|=m+1}\left|f_{\alpha}(y)\right|\right)\left(\sum_{k=1}^{n}\left|\left(x_{2}-x_{3}\right)_{k}\right|\right) \sum_{|\beta+\gamma|=m}\left|\frac{1}{\beta ! \gamma !}\left(x_{1}-x_{2}\right)^{\beta}\left(x_{2}-x_{3}\right)^{\gamma}\right| \\
& \leq\left(\sup _{y \in l,|\alpha|=m+1}\left|f_{\alpha}(y)\right|\right) \sqrt{n}\left|x_{2}-x_{3}\right| \frac{(\sqrt{n})^{m}}{m !}\left(\left|x_{1}-x_{2}\right|+\left|x_{2}-x_{3}\right|\right)^{m} \\
& \leq \frac{(\sqrt{n})^{m+1}|l|^{m}}{m !}\left(\sup _{y \in l,|\alpha|=m+1}\left|f_{\alpha}(y)\right|\right)\left|x_{2}-x_{3}\right| .
\end{aligned}
$$

Since $l$ is compact, we may assume that $A$ is compact. Then, by the definition of $\phi W_{A}^{*}$, there exists a stratification $\left\{A_{\tau}\right\}_{\tau}$ of $A$ such that for any $\tau$ we have $j_{\bar{A}_{\tau}, A}(F) \in W_{\bar{A}_{\tau}}^{*}(X)$. It follows from Theorem 1.1 .3 that, for any $\tau$, there exists a constant $C_{\tau}$ such that

$$
\left|R_{m-|\beta|+1}\left(S_{\beta} F ; x_{1}, x_{2}\right)\right| \leq C_{\tau}\left|x_{1}-x_{2}\right|^{m-|\beta|+2}
$$

holds for any $0 \leq|\beta| \leq m$ and $x_{1} x_{2} \in \bar{A}_{\tau}$. As the number of strata is finite, it makes sense to set

$$
C:=\max _{\tau} C_{\tau}
$$

Remark that the constant $C$ depends on $l, m, F$ and $A$ and it does not depend on $x_{1}$ and $x_{2}$. Now, the second term is estimated in the following way. If $x_{2}, x_{3} \in l$ belong to the closure of a same stratum, then

$$
\begin{aligned}
\sum_{|\beta| \leq m} \mid \frac{R_{m-|\beta|+1}\left(S_{\beta} F ; x_{2}, x_{3}\right)}{\beta !}( & \left.x_{1}-x_{2}\right)^{\beta}\left|\leq C \sum_{|\beta| \leq m}\right| x_{2}-\left.x_{3}\right|^{m-|\beta|+2} \frac{\left|x_{1}-x_{2}\right|^{|\beta|}}{\beta !} \\
& \leq C\left|x_{2}-x_{3}\right|^{2} \sum_{|\beta| \leq m}\left|x_{2}-x_{3}\right|^{m-|\beta|} \frac{\left|x_{1}-x_{2}\right|^{|\beta|}}{\beta !} \\
& \leq C\left|x_{2}-x_{3}\right|^{2}|l|^{m} \sum \frac{1}{\beta !} \\
& \leq e^{n} C|l|^{m}\left|x_{2}-x_{3}\right|^{2} .
\end{aligned}
$$

Now we take points $x=x_{0}, x_{1}, \ldots, x_{k}=x^{\prime}$ sequentially in the curve $l$ so that each pair $x_{i}$ and $x_{i+1}$ belong to the closure of a same stratum $(0 \leq i \leq k-1)$. 
Then

$$
\begin{aligned}
& \left|f_{0}(x)-T_{m}\left(F ; x, x^{\prime}\right)\right| \\
& \leq\left|T_{m}\left(F ; x_{0}, x_{0}\right)-T_{m}\left(F ; x_{0}, x_{1}\right)\right|+\left|T_{m}\left(F ; x_{0}, x_{1}\right)-T_{m}\left(F ; x_{0}, x_{2}\right)\right| \\
& \quad+\cdots+\left|T_{m}\left(F ; x_{0}, x_{k-1}\right)-T_{m}\left(F ; x_{0}, x_{k}\right)\right| \\
& \leq \frac{(\sqrt{n})^{m+1}|l|^{m}}{m !}\left(\sup _{y \in l,|\alpha|=m+1}\left|f_{\alpha}(y)\right|\right) \sum_{i=0}^{k-1}\left|x_{i}-x_{i+1}\right| \\
& \quad+e^{n} C|l|^{m} \sup _{0 \leq i \leq k-1}\left|x_{i}-x_{i+1}\right| \sum_{i=0}^{k-1}\left|x_{i}-x_{i+1}\right| .
\end{aligned}
$$

When $k$ tends to $\infty$, then the first term in the right hand side converges to

$$
\frac{(\sqrt{n}|l|)^{m+1}}{m !}\left(\sup _{y \in l,|\alpha|=m+1}\left|f_{\alpha}(y)\right|\right)
$$

and the second term tends to 0 .

The conclusion follows.

Corollary 2.2.6. - Let $X=\mathbb{R}^{n}$ and $A$ a locally closed 1-regular subanalytic subset in $X$. Then for any subanalytic open set $V \subset X$ and any compact subanalytic set $K \subset V$ there exists $\kappa>0$ such that, for any $h>0$, there exists $C_{h}>0$ satisfying

$$
\|F\|_{K \cap A, s, \kappa h}^{W} \leq C_{h}\|F\|_{\bar{V} \cap A, s, h}
$$

for any $F \in \varnothing W_{A}^{*}(X)$.

Proof. - By the definition of 1-regular, there exist a constant $M>0$ and a finite family $\left\{V_{i}\right\}$ of open subsets in $X$ such that $K \subset \cup V_{i} \subset V$ and, for any $i$ and $x_{1}, x_{2} \in V_{i} \cap A$, there exists a curve $l \subset A \cap V$ joining $x_{1}$ and $x_{2}$ satisfying $|l| \leq M\left|x_{1}-x_{2}\right|$.

Then, there exists a positive constant $\delta>0$ such that for any $x, y \in K$ with $|x-y|<\delta$, there exists $i$ such that $x, y \in V_{i}$.

First, assume that $x_{1}, x_{2} \in K \cap A$ satisfy $\left|x_{1}-x_{2}\right|<\delta$. Then, there exists a path $l \subset K \cap A$ joining $x_{1}$ and $x_{2}$ such that $|l| \leq M\left|x_{1}-x_{2}\right|$. Then, Lemma 2.2.5 implies that

$$
\begin{aligned}
\left|R_{m}\left(S_{\beta} F ; x_{1}, x_{2}\right)\right| & \leq \frac{(\sqrt{n}|l|)^{m+1}}{m !} \max _{|\alpha|=|\beta|+m+1} \sup _{y \in l}\left|f_{\alpha}(y)\right| \\
& \leq \frac{(\sqrt{n} M)^{m+1}}{m !}(|\beta|+m+1) !^{s} h^{|\beta|+m+1}|| F||_{\bar{V} \cap A, s, h}\left|x_{1}-x_{2}\right|^{m+1} .
\end{aligned}
$$

Moreover, we can suppose $\sqrt{n} M \geq 1$. Hence, if $|x-y| \leq \delta$, we obtain

$$
\|F\|_{K \cap A, s, \sqrt{n} M h}^{W} \leq\|F\|_{\bar{V} \cap A, s, h} .
$$


Now, assume that $x_{1}, x_{2} \in A \cap K$ satisfy $\left|x_{1}-x_{2}\right| \geq \delta$. For $h^{\prime}=(n+1) h$, we have

$$
\begin{aligned}
& \frac{m !\left|R_{m}\left(S_{\beta} F ; x_{1}, x_{2}\right)\right|}{h^{\prime|\beta|+m+1}(|\beta|+m+1) ! s\left|x_{1}-x_{2}\right|^{m+1}} \leq \frac{m !\left(\left|f_{\beta}\left(x_{1}\right)\right|+\left|T_{m}\left(S_{\beta} F ; x_{1}, x_{2}\right)\right|\right)}{h^{\prime|\beta|+m+1}(|\beta|+m+1) !^{s}\left|x_{1}-x_{2}\right|^{m+1}} \\
& \leq 2|| F \|_{\bar{V}, s, h} \sum_{|\gamma| \leq m} \frac{m !|\beta+\gamma|^{s} h^{|\beta+\gamma|}\left|x_{1}-x_{2}\right|^{|\gamma|}}{h^{\prime|\beta|+m+1}(|\beta|+m+1) !^{s} \gamma !\left|x_{1}-x_{2}\right|^{m+1}} \\
& \leq 2|| F \|_{\bar{V}, s, h} \frac{1}{h^{\prime}\left|x_{1}-x_{2}\right|} \frac{1}{(n+1)^{m}} \cdot \\
& \quad \cdot \sum_{|\gamma| \leq m} \frac{m !}{(m-|\gamma|) ! \gamma !} \frac{1}{(m-|\gamma|) !^{s-1}\left(h\left|x_{1}-x_{2}\right|\right)^{m-|\gamma|}} \\
& \leq 2|| F \|_{\bar{V}, s, h} \frac{1}{h^{\prime} \delta} \exp \left(\sigma(h \delta)^{-\sigma}\right) \frac{1}{(n+1)^{m}} \sum_{|\gamma| \leq m} \frac{m !}{(m-|\gamma|) ! \gamma !} \\
& =\frac{2}{h^{\prime} \delta} \exp \left(\sigma(h \delta)^{-\sigma}\right)|| F \|_{\bar{V}, s, h}
\end{aligned}
$$

where $\sigma=\frac{1}{s-1}$. The conclusion follows.

Let $X=\mathbb{R}^{n}, A \subset X$ a compact subanalytic set and $F \in \varnothing W_{A}^{*}(X)$. Since $A$ is compact, the number of strata of a stratification of $A$ is finite. Hence for any $h>0$ (resp. some $h>0)$ we have $\|F\|_{A, s, h}<\infty$ if $*=(s)($ resp. $*=\{s\})$ respectively. Set

$$
\delta W_{A, h}^{*}(X):=\left\{F \in \phi W_{A}^{*}(X) ;\|F\|_{A, s, h}<\infty\right\},
$$

and endow $\delta W_{A, h}^{*}(X)$ with the topology induced by the norm $\|\cdot\|_{A, s, h}$. Then, algebraically, we have

$$
\delta W_{A}^{(s)}(X) \simeq \lim _{h>0} \phi W_{A, h}^{(s)}(X)
$$

and

$$
\delta W_{A}^{\{s\}}(X) \simeq \underset{h>0}{\lim _{\longrightarrow}} \delta W_{A, h}^{\{s\}}(X) .
$$

Therefore, $\& W_{A}^{*}(X)$ can be endowed with a locally convex topology induced by these projective or inductive limits.

Proposition 2.2.7. - Let $X=\mathbb{R}^{n}$ and $A$ a 1-regular compact subanalytic subset in $X$. Then

$$
W_{A}^{*}(X)=\phi W_{A}^{*}(X) .
$$

Moreover, these spaces are topologically isomorphic. In particular, $8 W_{A}^{*}(X)$ is an FS space if $*=(s)$, and a DFS space if $*=\{s\}$. 
Proof. - In Corollary 2.2.6, choose $K$ as $A$ and $V$ as an open subanalytic subset containing $A$. Then we have

$$
\|F\|_{A, s, \kappa h}^{W} \leq C\|F\|_{A, s, h}
$$

The result follows.

Proposition 2.2.8. - Let $X$ be a real analytic manifold and $A$ a locally closed subanalytic set. If $A$ is 1-regular at $p \in X$, then $W_{A, p}^{*}=8 W_{A, p}^{*}$.

Proof. - Note that for any $p \in X$ we have

$$
W_{A, p}^{*}=\underset{p \in U}{\lim } W_{A \cap \bar{U}}^{*}(X), \quad \phi W_{A, p}^{*}=\underset{p \in U}{\lim } \phi W_{A \cap \bar{U}}^{*}(X) .
$$

Further, we have

$$
W_{A \cap \bar{U}}^{*}(X) \subset \varnothing W_{A \cap \bar{U}}^{*}(X) .
$$

On the other hand, for any sufficiently small open subanalytic neighborhoods $U_{1} \supset U_{2} \supset U_{3}$ of $p$, Corollary 2.2.6 implies that the restriction map $\delta W_{A \cap \bar{U}_{1}}^{*}(X) \rightarrow \phi W_{A \cap \bar{U}_{3}}^{*}(X)$ factorizes through $W_{A \cap \bar{U}_{2}}^{*}(X)$. Hence the following diagram commutes

$$
\begin{aligned}
\delta W_{A \cap \bar{U}_{1}}^{*}(X) \longrightarrow & W_{A \cap \bar{U}_{2}}^{*}(X) \\
\searrow & \downarrow \\
& \delta W_{A \cap \bar{U}_{3}}^{*}(X) .
\end{aligned}
$$

The conclusion follows.

Corollary 2.2.9. - Let $X$ be a real analytic manifold, $A \subset X$ a locally closed subanalytic set and $\left\{A_{\alpha}\right\}$ a 1-regular stratification of $A$. Then, $\phi W_{A}^{*}=$ $\phi W_{\left\{A_{\alpha}\right\}}^{*}$.

Proof. - The result comes from the fact that for any stratum $A_{\alpha}$, we have

$$
j_{A_{\alpha}, A}\left(\varnothing W_{A}^{*}\right) \subset \varnothing W_{A_{\alpha}}^{*}=W_{A_{\alpha}}^{*} .
$$

Corollary 2.2.10. - Let $X$ be a real analytic manifold, and let $A_{1}$ and $A_{2}$ be closed subanalytic subsets, or open subanalytic subsets in $X$. If $A_{1} \cap A_{2}$ is 1-regular at $p \in X$, then the sequence

$$
0 \rightarrow \phi W_{A_{1} \cup A_{2}, p}^{*} \rightarrow \phi W_{A_{1}, p}^{*} \oplus \phi W_{A_{2}, p}^{*} \rightarrow \phi W_{A_{1} \cap A_{2}, p}^{*} \rightarrow 0
$$

is exact.

Proof. - By Lemma 2.2.4, it is sufficient to prove the surjectivity. Since $\varnothing W_{A_{1} \cap A_{2}}^{*}=W_{A_{1} \cap A_{2}}^{*}$ holds, the result is clear. 
The condition " $A_{1} \cap A_{2}$ is 1-regular" in Corollary 2.2.10 is too strong. Indeed, if $\operatorname{dim} X=2$, and if $A_{1}$ and $A_{2}$ are closed subanalytic subsets, then (2.6) is always exact, see Theorem 3.1.4. We also note that, in the case of $\operatorname{dim} X>2$, we can find an example in which the surjectivity of the third morphism of (2.6) does not hold.

Lemma 2.2.11. - Let $X$ be a real analytic manifold and $A \subset X$ a compact subanalytic set. Then $\delta W_{A}^{(s)}(X)$ (resp. $\left.\delta W_{A}^{\{s\}}(X)\right)$ can be endowed with an FS (resp. a DFS) locally convex topology. Moreover if $A$ is a finite union of compact subanalytic sets $B_{1}, \ldots, B_{k}$, then the canonical morphism

$$
\iota: \phi W_{A}^{*}(X) \rightarrow \underset{1 \leq i \leq k}{\oplus} \phi W_{B_{i}}^{*}(X)
$$

gives a topological isomorphism onto its image.

Proof. - Let $\left\{A_{i}\right\}$ be a 1-regular stratification of $A$ satisfying the following condition. For each stratum $A_{i}$, there exist an open subset $U \subset X$ containing $\bar{A}_{i}$ and isomorphism $\varphi_{i}: U \rightarrow V$ for some open subset $V$ in $\mathbb{R}^{n}$.

It follows from Lemma 2.2.4 that the following sequence is exact,

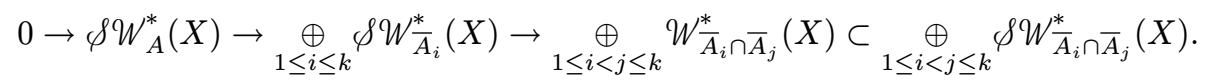

We can consider the sets $\bar{A}_{i}$ as 1 -regular compact subanalytic subsets of $\mathbb{R}^{n}$. Hence $\& W_{A_{i}}^{*}(X)$ has an FS or a DFS locally convex topology by Proposition 2.2.7, and the morphism

$$
\underset{1 \leq i \leq k}{\oplus} \phi W \bar{A}_{i}^{*}(X)=\underset{1 \leq i \leq k}{\oplus} W_{\bar{A}_{i}}^{*}(X) \rightarrow \underset{1 \leq i<j \leq k}{\oplus} W_{\bar{A}_{i} \cap \bar{A}_{j}}(X)
$$

is continuous for such topologies. We endow $\& W_{A}^{*}(X)$ with the induced topology. By the exactness of the above sequence, the topological space $\delta W_{A}^{*}(X)$ is a closed subspace of $\underset{1 \leq i \leq k}{\oplus} \phi W_{A_{i}}^{*}(X)$. Therefore $\phi W_{A}^{*}(X)$ is an FS or a DFS space.

One can check that another choice of 1-regular stratifications and morphisms $\varphi_{i}$ induces an equivalent topology. Indeed, by considering a 1-regular stratification finer than those, we can reduce the problem to the following claim. Let $A \subset \mathbb{R}^{n}$ be a compact 1-regular subanalytic subset and $A_{i} \subset A\left(\subset \mathbb{R}^{n}\right)$ $(i=1,2, \ldots, k)$ compact 1 -regular subanalytic subsets with $A=\cup A_{i}$. Then the canonical morphism

$$
\iota: \phi W_{A}^{*}(X) \rightarrow \underset{1 \leq i \leq k}{\oplus} \phi W_{A_{i}}^{*}(X)
$$

is a topological isomorphism onto its image.

If $*=(s)$, then these vector spaces have FS topologies and the image of $\iota$ is closed by Lemma 2.2.4. Hence the claim follows from the open mapping theorem. 
Now, let us prove the claim for $*=\{s\}$. Since a DFS space is bornological, it suffices to show that for a sequence $\left\{x_{j}\right\}_{j \in \mathbb{N}} \subset \delta W_{A}^{\{s\}}(X)$ with $\iota\left(x_{j}\right) \rightarrow 0(j \rightarrow$ $\infty)$, the sequence $\left\{x_{j}\right\}$ also tends to 0 . Since $\left\{\iota\left(x_{j}\right)\right\}$ is bounded in the DFS space $\underset{1<i \leq k}{\oplus} \delta W_{A_{i}}^{\{s\}}(X)$, there exists an $h>0$ with $\iota\left(x_{j}\right) \in \underset{1 \leq i \leq k}{\oplus} \delta W_{A_{i}, h}^{\{s\}}(X)$ for any $j$. Then, as $A=\cup A_{i}$, we have the estimate

$$
\|x\|_{A, s, h} \leq \sum_{i=0}^{k}\|\iota(x)\|_{A_{i}, s, h}
$$

and from which the claim follows.

The last assertion in the lemma can be proved in the similar way.

If $X=\mathbb{R}^{n}, A \subset X$ a compact subanalytic set and $*=(s)$, the FS topology in $\phi W_{A}^{(s)}(X)$ is described as follows. Given a sequence $\left\{F_{n}\right\} \subset \& W_{A}^{(s)}(X)$, one has that

$$
\lim _{n \rightarrow \infty} F_{n} \rightarrow 0 \Longleftrightarrow\left\|F_{n}\right\|_{A, s, h} \rightarrow 0 \text { for any } h>0 .
$$

Note that the convergence in $W_{A}^{(s)}(X)$ is defined in the following way. Given a sequence $\left\{G_{n}\right\} \subset W_{A}^{(s)}(X)$,

$$
\lim _{n \rightarrow \infty} G_{n} \rightarrow 0 \Longleftrightarrow\left\|G_{n}\right\|_{A, s, h} \rightarrow 0 \text { and }\left\|G_{n}\right\|_{A, s, h}^{W} \rightarrow 0 \text { for any } h>0 .
$$

These two topologies coincides if $A$ is 1-regular.

2.3. The sheaf of the stratified Whitney jets on the subanalytic site. - Let $X$ be a real analytic manifold. The subanalytic presheaf of stratified Whitney jets of class $*$ is defined by

$$
\phi W_{X_{\mathrm{sa}}}^{*}(U):=\phi W_{U}^{*}(X)
$$

where $U$ is a subanalytic open subset of $X$.

TheOREM 2.3.1. - The presheaf $8 W_{X_{\mathrm{sa}}}^{*}$ is a sheaf on $X_{\mathrm{sa}}$ and a $\varrho_{!} \mathscr{D}_{X^{-}}$ module.

Proof. - It follows from Lemma 2.2.4 and the obvious fact that $\Gamma\left(\bar{U}, \mathscr{D}_{X}\right)$ acts on $\phi W_{U}^{*}(X)$.

Proposition 2.3.2. - If $U \subset X$ is a 1-regular open subanalytic set, then

$$
\phi W_{X_{\mathrm{sa}}}^{*}(U)=W_{U}^{*}(X) \simeq W_{\bar{U}}^{*}(X) \simeq \frac{\mathscr{C}^{*}(X)}{\mathcal{J}_{X, \bar{U}}^{*}(X)},
$$

where $\mathcal{I}_{X, \bar{U}}^{*}$ denotes the subsheaf of $\mathscr{C}^{*}$ consisting of functions vanishing on $\bar{U}$ up to infinite order.

Corollary 2.3.3. - For $U \in \mathrm{Op}^{c}\left(X_{\mathrm{sa}}\right)$, set $W_{X_{\mathrm{sa}}}^{*}(U):=W_{U}^{*}(X)$. Then $W_{X_{\mathrm{sa}}}^{* a} \simeq \phi W_{X_{\mathrm{sa}}}^{*}$. 
Proof. - It is sufficient to combine Proposition 2.3.2, Lemma 2.1.5 and Proposition 2.1.4.

2.4. Stratified ultradistributions. - Let $X$ be a real analytic manifold, $A$ a closed subanalytic subset in $X$ and let $\mathscr{D} b^{*}$ denote the sheaf of ultradistributions of class $*$. For any stratification $\left\{A_{\alpha}\right\}$ of $A$, let us define stratified ultradistributions along $\left\{A_{\alpha}\right\}$.

Definition 2.4.1. - An ultradistribution $u \in \mathscr{D} b^{*}(U)$ is said to be stratified along $\left\{A_{\alpha}\right\}$ in $U$ if $u$ can be written in the form:

$$
u=\sum_{\alpha} u_{\alpha}, \quad u_{\alpha} \in \Gamma_{\bar{A}_{\alpha}}\left(U, D b^{*}\right) .
$$

We define the sheaf of stratified ultradistributions of class $*$ along $\left\{A_{\alpha}\right\}$ as

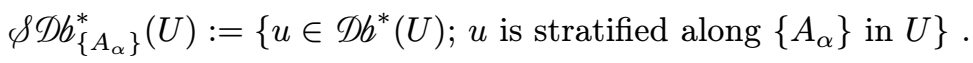

For a stratification $\left\{A_{\alpha}^{\prime}\right\}$ finer than $\left\{A_{\alpha}\right\}$, there exists the canonical morphism

$$
\& \mathscr{D} b_{\left\{A_{\alpha}^{\prime}\right\}}^{*}(U) \rightarrow \varnothing \mathscr{D} b_{\left\{A_{\alpha}\right\}}^{*}(U) .
$$

We define the sheaf of stratified ultradistributions of class $*$ along $A$ as

$$
\phi \mathscr{D} b_{[A]}^{*}(U):=\underbrace{}_{\substack{\text { stratification } \\\left\{A_{\alpha}\right\} \text { of } A}} \phi \mathscr{D} b_{\left\{A_{\alpha}\right\}}^{*}(U) .
$$

Since for any stratification $\left\{A_{\alpha}\right\}$ there exists a 1-regular stratification finer than $\left\{A_{\alpha}\right\}$, we have

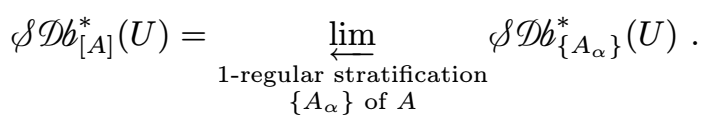

There exists the canonical injective sheaf homomorphism

$$
\& \mathscr{D} b_{[A]}^{*} \hookrightarrow \Gamma_{A}\left(\mathscr{D} b^{*}\right) .
$$

This morphism is not surjective in general. The following lemma follows easily from the definition.

Lemma 2.4.2. - Let $X$ be a real analytic manifold, and let $A_{1}, \ldots, A_{l}$ be closed subanalytic subsets in $X$. Then the sheaf homomorphism

$$
\oplus \varnothing \mathscr{D} b_{\left[A_{i}\right]}^{*} \rightarrow \varnothing \mathscr{D} b_{\left[\cup A_{i}\right]}^{*}
$$

is surjective.

Remark that, in general, the middle of the sequence

$$
0 \rightarrow \varnothing \mathscr{D} b_{\left[A_{1} \cap A_{2}\right]}^{*} \rightarrow \varnothing \mathscr{D} b_{\left[A_{1}\right]}^{*} \oplus \varnothing \mathscr{D} b_{\left[A_{2}\right]}^{*} \rightarrow \varnothing \mathscr{D} b_{\left[A_{1} \cup A_{2}\right]}^{*} \rightarrow 0
$$

is not exact. 
THEOREM 2.4.3. - Let $X$ be a real analytic manifold, and $A \subset X$ a compact subanalytic set. Then, algebraically, we have

$$
\phi \mathscr{D} b_{[A]}^{*}(X) \simeq\left(\phi W_{A}^{*} \underset{G}{\otimes} V_{X}(X)\right)^{\prime}
$$

where $\left(\phi W_{A}^{*} \underset{\mathscr{Q}}{\otimes V_{X}}(X)\right)^{\prime}$ denotes the topological dual space of $\phi W_{A}^{*} \underset{\mathscr{G}}{\otimes V_{X}}(X)$ and $V_{X}$ designates the sheaf of volume elements in $X$, i.e., $\omega_{X}^{(n)} \underset{\mathbb{Z}}{\otimes}$ or $r_{X}$.

Proof. - The continuous morphism $j_{A}: \mathscr{C}^{*}(X) \rightarrow \phi W_{A}^{*}(X)$ induces the morphism

$$
j_{A}^{t}:\left(\varnothing W_{A}^{*} \underset{\mathscr{Q}}{\otimes} V_{X}(X)\right)^{\prime} \rightarrow\left(\mathscr{C}^{*} \underset{\mathscr{Q}}{\otimes} V_{X}(X)\right)^{\prime} \subset \mathscr{D} b^{*}(X) .
$$

For any $\varphi(x) \in \mathscr{C}^{*}(X)$ with $\operatorname{supp}(\varphi) \cap A=\varnothing$, we have $j_{A}(\varphi(x))=0$. Hence $\operatorname{im} j_{A}^{t} \subset \Gamma_{A}\left(X, \mathscr{D} b^{*}\right)$. Moreover since $j_{A}\left(\mathscr{C}^{*}(X)\right)$ is dense in $8 W_{A}^{*}(X)$ by Proposition A.7, the morphism

$$
j_{A}^{t}:\left(\varnothing W_{A}^{*} \underset{\mathscr{Q}}{\otimes} V_{X}(X)\right)^{\prime} \hookrightarrow \Gamma_{A}\left(X, \mathscr{D} b^{*}\right)
$$

is injective.

Let $A_{1}, A_{2}, \ldots, A_{l}$ be closed subanalytic subsets in $X$ with $\cup A_{i}=A$. If we prove that, for each $i$,

$$
j_{A_{i}}^{t}\left(\left(\& W_{A_{i}}^{*} \underset{\mathscr{G}}{\otimes V_{X}}(X)\right)^{\prime}\right)=\varnothing \mathscr{D} b_{\left[A_{i}\right]}^{*}(X),
$$

then

$$
j_{A}^{t}\left(\left(\varnothing W_{A}^{*} \underset{G}{\otimes} V_{X}(X)\right)^{\prime}\right)=\varnothing \mathscr{D} b_{[A]}^{*}(X)
$$

follows from the following commutative diagram

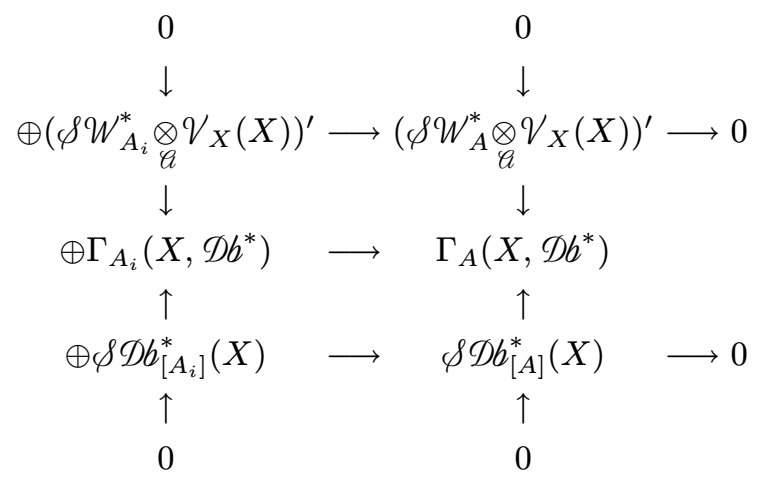

The first row of (2.7) is exact since

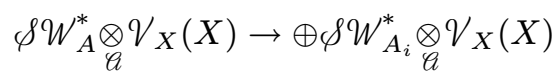


is an injective homomorphism of locally topological vector spaces by Lemma 2.2.11. The third row of (2.7) is exact by Lemma 2.4.2. All vertical arrows of (2.7) are injective.

By these observations, we can reduce the problem to the case $X=\mathbb{R}^{n}$ and $A \subset X$ is a compact subanalytic set. First recall that if $B$ is a compact set in $\mathbb{R}^{n}$, then it follows from the result of Whitney and Kantor that

$$
j_{B}^{t}:\left(W_{B}^{*}(X)\right)^{\prime} \simeq \Gamma_{B}\left(X, D b^{*}\right) .
$$

Let $\left\{A_{\alpha}\right\}$ be a 1-regular stratification of $A$. Let us consider the following commutative diagram

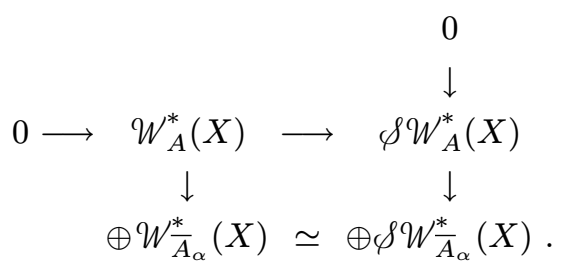

Here the first horizontal arrow is injective and has a dense image by Proposition A.7. Since each $\bar{A}_{\alpha}$ is 1-regular, the second horizontal arrow is topologically isomorphism. The second vertical arrow is a topological isomorphism onto its image. Then taking the dual of the diagram, we have

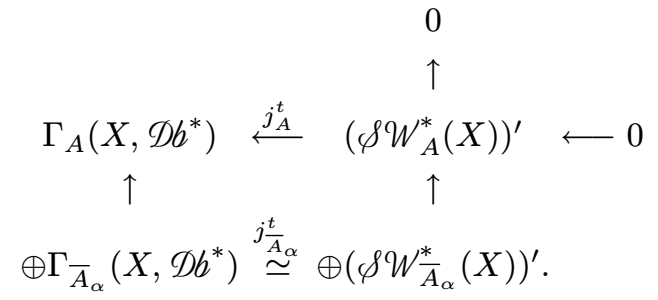

Hence we can conclude

$$
j_{A}^{t}\left(\left(\varnothing W_{A}^{*}(X)\right)^{\prime}\right)=\operatorname{Im}\left(\oplus \Gamma_{\bar{A}_{\alpha}}\left(X, \mathscr{D} b^{*}\right)\right)=\varnothing \mathscr{D} b_{\left\{A_{\alpha}\right\}}^{*}(X)
$$

for any 1-regular stratification $\left\{A_{\alpha}\right\}$ of $A$. In particular, we obtain

$$
j_{A}^{t}\left(\left(\varnothing W_{A}^{*}(X)\right)^{\prime}\right)=\lim _{\longleftarrow} \varnothing \mathscr{D} b_{\left\{A_{\alpha}\right\}}^{*}(X)=\varnothing \mathscr{D} b_{[A]}^{*}(X) .
$$

The conclusion follows.

Corollary 2.4.4. - Let $X$ be a real analytic manifold, $A \subset X$ a closed subanalytic set. If $\left\{A_{\alpha}\right\}$ is a 1-regular stratification of $A$, then we have

$$
\varnothing \mathscr{D} b_{[A]}^{*}=\varnothing \mathscr{D} b_{\left\{A_{\alpha}\right\}}^{*} .
$$

In particular, if $A$ is 1-regular at $p \in X$, then we have

$$
\left(\varnothing \mathscr{D} b_{[A]}^{*}\right)_{p}=\left(\Gamma_{A}\left(\mathscr{D} b^{*}\right)\right)_{p} .
$$

TOME $139-2011-\mathrm{N}^{\mathrm{O}} 3$ 
Proof. - Set $Z\left(A_{\beta}\right):=\bar{A}_{\gamma} \cap \bar{A}_{\beta} \neq \varnothing \quad \bar{A}_{\gamma}$. For any $p \in A_{\beta}$, since $Z\left(A_{\beta}\right)$ is a closed neighborhood of $p$ in $A$, we have

$$
\left(\& \mathscr{D} b_{\left\{A_{\alpha}\right\}}^{*}\right)_{p}=\left(\& \mathscr{D} b_{\left\{A_{\alpha} \cap Z\left(A_{\beta}\right)\right\}}^{*}\right)_{p}, \quad\left(\& \mathscr{D} b_{[A]}^{*}\right)_{p}=\left(\& \mathscr{D} b_{\left[A \cap Z\left(A_{\beta}\right)\right]}^{*}\right)_{p} .
$$

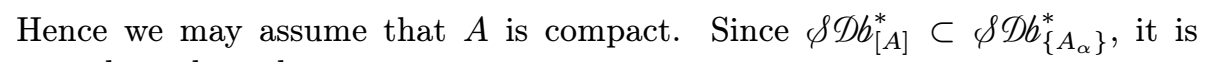
enough to show that

$$
\left(\phi \mathscr{D} b_{[A]}^{*}\right)_{p} \hookrightarrow\left(\& D b_{\left\{A_{\alpha}\right\}}^{*}\right)_{p}
$$

is surjective. By the softness of the sheaf $\& \mathscr{D} b_{\left\{A_{\alpha}\right\}}^{*}$,

$$
\phi \mathscr{D} b_{\left\{A_{\alpha}\right\}}^{*}(X) \rightarrow\left(\& \mathscr{D} b_{\left\{A_{\alpha}\right\}}^{*}\right)_{p}
$$

is surjective. Hence it is sufficient to show $\phi \mathscr{D} b_{\left\{A_{\alpha}\right\}}^{*}(X)=\varnothing \mathscr{D} b_{[A]}^{*}(X)$. This follows from the equations (2.8) and (2.9).

Corollary 2.4.5. - Let $X$ be a real analytic manifold, $A_{1}, A_{2} \subset X$ closed subanalytic sets. If $A_{1} \cap A_{2}$ is 1-regular at $p \in X$, then the sequence

$$
0 \rightarrow \varnothing \mathscr{D} b_{\left[A_{1} \cap A_{2}\right], p}^{*} \rightarrow \varnothing \mathscr{D} b_{\left[A_{1}\right], p}^{*} \oplus \varnothing \mathscr{D} b_{\left[A_{2}\right], p}^{*} \rightarrow \varnothing \mathscr{D} b_{\left[A_{1} \cup A_{2}\right], p}^{*} \rightarrow 0
$$

is exact.

Proof. — The injectivity is clear, and the surjectivity comes from Lemma 2.4.2. The exactness of the middle follows from

$$
\phi \mathscr{D} b_{\left[A_{1}\right]}^{*} \cap \mathscr{D D} b_{\left[A_{2}\right]}^{*} \subset \Gamma_{A_{1} \cap A_{2}}\left(\mathscr{D} b^{*}\right)
$$

and $\Gamma_{A_{1} \cap A_{2}}\left(\mathscr{D} b^{*}\right)=\varnothing \mathscr{D} b_{\left[A_{1} \cap A_{2}\right]}^{*}$ at $p$.

Similarly to the exactness of (2.6), the exactness of (2.10) holds if $\operatorname{dim} X \leq 2$ without the assumption of 1-regularity on $A_{1} \cap A_{2}$.

If $A$ is a compact subanalytic set, then $\& \mathscr{D} b_{[A]}^{*}(X)$ is equipped with the strong dual topology of the locally convex topological vector space $\delta W_{A}^{*}(X)$. Then $\& \mathscr{D} b_{[A]}^{*}(X)$ is a DFS (resp. an FS) space if $*=(s)$ (resp. $*=\{s\}$ ) respectively. Since $\varnothing \mathscr{D} b_{[A]}^{*}(X)$ and $\Gamma_{A}\left(X, \mathscr{D} b^{*}\right)$ are reflexive, $\varnothing \mathscr{D} b_{[A]}^{*}(X)$ is dense in $\Gamma_{A}\left(X, \mathscr{D} b^{*}\right)$.

\section{Sheaves on subanalytic sites relative to real surfaces}

In this section we are going to study in detail the extension properties of stratified Whitney jets on real surfaces. 
3.1. On the exactnesses of (2.6) and (2.10) in dimension 2. - Throughtout the subsection $X$ is a real analytic manifold of dimension 2, unless otherwise specified.

Definition 3.1.1. - Let $A \subset X$ be a closed subanalytic set. We say that a 1regular stratification $\left\{A_{\alpha}\right\}$ of $A$ is good if every $\bar{A}_{\alpha}$ is topologically isomorphic to $D^{\operatorname{dim}_{\mathbb{R}} A_{\alpha}}$ as a topological manifold with the boundary, where $D^{k}$ denotes a closed unit disc in $\mathbb{R}^{k}$.

By [10], for any stratification $\left\{A_{\alpha}\right\}$ of $A$, there exists a good 1-regular stratification finer than $\left\{A_{\alpha}\right\}$.

Lemma 3.1.2. - Let $A \subset X$ be a closed subanalytic set, $\left\{A_{\alpha}\right\}$ a good 1-regular stratification of $A$. For any $A_{\alpha}$ with $\operatorname{dim} A_{\alpha}=2$, the restriction map

$$
\delta W_{X \backslash A_{\alpha}}^{*}(X) \rightarrow \& W_{\bar{A}_{\alpha} \backslash A_{\alpha}}^{*}(X)
$$

is surjective.

Proof. - Let $A_{\alpha}$ satisfy $\operatorname{dim} A_{\alpha}=2$. Set $Z:=\bar{A}_{\alpha} \backslash A_{\alpha}$ and let $\left\{Z_{\beta}\right\}$ be the induced good 1-regular stratification of $Z$. For any $p$ and $\epsilon>0, D_{\epsilon}(p)$ designates the closed disk with center $p$ and radius $\epsilon$. By the partition of unity, it is enough to show that for any $p \in Z$, there exists $\epsilon>0$ such that

$$
\delta W_{D_{\epsilon}(p) \backslash A_{\alpha}}^{*}(X) \rightarrow \varnothing W_{Z \cap D_{\epsilon}(p)}^{*}(X)
$$

is surjective.

If $p \in Z_{\beta}$ with $\operatorname{dim} Z_{\beta}=1$, then $Z \cap D_{\epsilon}(p)$ is 1-regular for sufficiently small $\epsilon>0$. The result is clear in this case.

Suppose, now, $Z_{\beta}=\{p\}$. Since $Z$ is topologically trivial, there exist only two strata $Z_{1}$ and $Z_{2}$ such that $\operatorname{dim} Z_{i}=1$ and $p \in \bar{Z}_{i}(i=1,2$,$) . Let \epsilon>0$ be such that $Z_{1}$ and $Z_{2}$ cross $\partial D_{\epsilon}(p)$ transversally and any stratum other than $Z_{1}, Z_{2}$ and $Z_{\beta}$ does not intersect with $D_{\epsilon}(p)$.

Since $\bar{A}_{\alpha}$ is 1-regular, the angle between the tangent lines of $Z_{1}$ and $Z_{2}$ at $p$ in the side of $D_{\epsilon}(p) \backslash A_{\alpha}$ is positive. Hence, if $\epsilon$ is sufficiently small, then there exists $q \in \partial D_{\epsilon}(p) \backslash A_{\alpha}$ such that the segment $l$ from $p$ to $q$ is contained in $D_{\epsilon}(p) \backslash A_{\alpha}$, and that $Z_{i}$ is not tangent to $l(i=1,2)$. One checks easily that $D_{\epsilon}^{\circ}(p) \backslash\left(l \cup \bar{Z}_{1} \cup \bar{Z}_{2}\right)$ has three connected components, one of whose is $\AA_{\alpha} \cap B_{\epsilon}(p)$. Denote by $W_{1}$ and $W_{2}$ the other two connected components. The sets $W_{1}$ and $W_{2}$ satisfy

1. $\bar{W}_{1} \cap \bar{W}_{2}=l$ and $\bar{W}_{1} \cup \bar{W}_{2}=D_{\epsilon}(p) \backslash A_{\alpha}$,

2. the boundary $\partial W_{i}$ of $W_{i}$ consists of $Z_{i}, l$ and a part of the circle, in particular, $W_{i}$ and $\partial W_{i}$ are 1-regular $(i=1,2)$.

TOME $139-2011-\mathrm{N}^{\mathrm{O}} 3$ 
Let $F \in \& W_{Z \cap D_{\epsilon}(p)}^{*}(X)$. For sake of simplicity, we assume that $\left.F\right|_{Z_{\beta}}=0$ and $\left.F\right|_{Z \cap \partial D_{\epsilon}(p)}=0$. Then, we define $F_{i} \in \delta W_{\partial W_{i}}^{*}(X)(i=1,2)$ by

$$
F_{i}(x):=\left\{\begin{array}{cl}
F(x) & \text { if } x \in Z_{i}, \\
0 & \text { if } x \notin Z_{i} .
\end{array}\right.
$$

Since $\partial W_{i}$ is 1-regular, we can find a function $\varphi_{i}(x) \in \mathscr{C}^{*}(X)$ such that $j_{\partial W_{i}}\left(\varphi_{i}\right)=F_{i}$. Noticing

$$
\left.j_{\bar{W}_{1}} \varphi_{1}\right|_{\bar{W}_{1} \cap \bar{W}_{2}}=\left.j_{\bar{W}_{2}} \varphi_{2}\right|_{\bar{W}_{1} \cap \bar{W}_{2}}=0
$$

the jet

$$
G(x):=\left\{\begin{array}{l}
j_{\bar{W}_{1}}\left(\varphi_{1}\right) x \in \bar{W}_{1} \\
j_{\bar{W}_{2}}\left(\varphi_{2}\right) x \in \bar{W}_{2}
\end{array}\right.
$$

belongs to $\phi W_{D_{\epsilon}(p) \backslash A_{\alpha}}^{*}(X)$, and $\left.G(x)\right|_{Z \cap D_{\epsilon}(p)}=F$.

By the similar arguments as in the proof of Lemma 3.1.2, we can also prove the following lemma.

Lemma 3.1.3. - Let $A \subset X$ be a closed subanalytic set, $\left\{A_{\alpha}\right\}$ a good 1-regular stratification of $A$. For any $A_{\alpha}$ with $\operatorname{dim} A_{\alpha}=2$, we have

$$
\& \mathscr{D} b_{\left[\bar{A}_{\alpha} \backslash A_{\alpha}\right]}^{*}(X)=\varnothing \mathscr{D} b_{\left[X \backslash A_{\alpha}\right]}^{*}(X) \cap \Gamma_{\bar{A}_{\alpha} \backslash A_{\alpha}}\left(X, \mathscr{D} b^{*}\right) .
$$

Theorem 3.1.4. - Let $Z_{1}, Z_{2} \subset X$ be closed subanalytic sets. The sequence

$$
0 \rightarrow \& W_{Z_{1} \cup Z_{2}}^{*} \rightarrow \phi W_{Z_{1}}^{*} \oplus \delta W_{Z_{2}}^{*} \rightarrow \phi W_{Z_{1} \cap Z_{2}}^{*} \rightarrow 0
$$

is exact.

Proof. - Since it is a local problem, we may assume that $X=\mathbb{R}^{2}$ and $Z_{i}$ is compact. Set $Z:=Z_{1} \cup Z_{2}$. Let $\left\{Z_{\alpha}\right\}_{\alpha \in \Lambda}$ be a good 1-regular stratification of $Z_{1} \cup Z_{2}$ finer than the partition $\left\{Z_{1} \cup Z_{2}, Z_{1}, Z_{2}, Z_{1} \cap Z_{2}\right\}$. Note that $\Lambda$ is a finite set.

We will prove the assertion by induction of the cardinality of $\Lambda$.

By Lemma 2.4.2, it is enough to show the exactness of the sequence

$$
\delta W_{Z_{1}}^{*}(X) \oplus \phi W_{Z_{2}}^{*}(X) \rightarrow \phi W_{Z_{1} \cap Z_{2}}^{*}(X) \rightarrow 0 .
$$

Let $\beta \in \Lambda$ be such that

$$
\operatorname{dim}\left(Z_{1} \cup Z_{2}\right)=\operatorname{dim} Z_{\beta} .
$$

For $i=1,2$, set

$$
Z_{i}^{\prime}:=Z_{i} \backslash Z_{\beta}
$$

Note that $Z_{i}^{\prime}$ is a closed subanalytic set and

$$
Z_{\beta} \subset Z_{i} \Longrightarrow Z_{i}^{\prime} \cap \bar{Z}_{\beta}=\partial Z_{\beta} \quad(i=1,2) .
$$

BULletin DE LA SOCIÉtÉ MATHÉMATIQUE DE FRANCE 
The sequence

$$
\phi W_{Z_{1}^{\prime}}^{*}(X) \oplus \phi W_{Z_{2}^{\prime}}^{*}(X) \rightarrow \phi W_{Z_{1}^{\prime} \cap Z_{2}^{\prime}}^{*}(X)=\varnothing W_{\left(Z_{1} \cap Z_{2}\right) \backslash Z_{\beta}}^{*}(X) \rightarrow 0
$$

is exact by the induction hypothesis.

Let $F \in \phi W_{Z_{1} \cap Z_{2}}^{*}(X)$. For sake of simplicity, we assume

$$
\left.F\right|_{\bar{Z}_{\beta}}=0 \text {. }
$$

It follows from the above exact sequence that there exist $F_{1} \in \phi W_{Z_{1}^{\prime}}^{*}(X), F_{2} \in$ $\phi W_{Z_{2}^{\prime}}^{*}(X)$ such that

$$
\left.F_{1}\right|_{\left(Z_{1} \cap Z_{2}\right) \backslash Z_{\beta}}-\left.F_{2}\right|_{\left(Z_{1} \cap Z_{2}\right) \backslash Z_{\beta}}=\left.F\right|_{\left(Z_{1} \cap Z_{2}\right) \backslash Z_{\beta}} .
$$

Suppose $Z_{\beta} \subset Z_{1}$. Then if $\operatorname{dim}\left(Z_{\beta}\right)=2$, by Lemma 3.1.2 there exists $\tilde{F} \in$ $\phi W_{\left(Z_{1} \cup Z_{2}\right) \backslash Z_{\beta}}^{*}(X)$ such that

$$
\left.\tilde{F}\right|_{\partial Z_{\beta}}=\left.F_{1}\right|_{\partial Z_{\beta}} \text {. }
$$

Moreover, if $\operatorname{dim} Z_{\beta}<2$, then $\partial Z_{\beta}$ consists of isolated points, hence there exists $\tilde{F} \in \phi W_{\left(Z_{1} \cup Z_{2}\right) \backslash Z_{\beta}}^{*}(X)$ such that $\left.\tilde{F}\right|_{\partial Z_{\beta}}=\left.F_{1}\right|_{\partial Z_{\beta}}$.

Set

$$
\tilde{F}_{1}:=F_{1}-\left.\tilde{F}\right|_{Z_{1}^{\prime}}, \quad \tilde{F}_{2}:=F_{2}-\left.\tilde{F}\right|_{Z_{2}^{\prime}} .
$$

Remark that

$$
\left.\tilde{F}_{1}\right|_{\left(Z_{1} \cap Z_{2}\right) \backslash Z_{\beta}}-\left.\tilde{F}_{2}\right|_{\left(Z_{1} \cap Z_{2}\right) \backslash Z_{\beta}}=\left.F\right|_{\left(Z_{1} \cap Z_{2}\right) \backslash Z_{\beta}} \text {. }
$$

Taking (3.2) and $\left.\tilde{F}_{1}\right|_{\partial Z_{\beta}}=0$ into account, we can extend $\tilde{F}_{1}$ to an element of $\phi W_{Z_{1}}^{*}(X)$ by zero (i.e. the zero extension). Now, if $Z_{\beta} \cap Z_{2}=\varnothing$, then $Z_{2}=Z_{2}^{\prime}$ and the result follows. Otherwise, suppose $Z_{\beta} \subset Z_{2}$, then, since $\left.\tilde{F}\right|_{\partial Z_{\beta}}=\left.\tilde{F}_{1}\right|_{\partial Z_{\beta}}=0$, we have $\left.\tilde{F}_{2}\right|_{\partial Z_{\beta}}=0$. Hence $\tilde{F}_{2}$ is regarded as an element in $\phi W_{Z_{2}}^{*}(X)$ by the zero extension.

The conclusion follows.

In general, for open subanalytic subsets $U_{1}$ and $U_{2}$, the sequence

$$
0 \rightarrow \phi W_{U_{1} \cup U_{2}}^{*} \rightarrow \phi W_{U_{1}}^{*} \oplus \phi W_{U_{2}}^{*} \rightarrow \phi W_{U_{1} \cap U_{2}}^{*} \rightarrow 0
$$

is not exact, indeed the surjectivity does not hold. The lack of surjectivity is of topological nature and it comes from the fact that $R \mathcal{H}$ om $\left(\mathbb{C}_{U_{1} \cup U_{2}}, \mathbb{C}_{X}\right)$ is not necessarily concentrated in degree 0 .

Corollary 3.1.5. - Let $Z_{1}, Z_{2} \subset X$ be closed subanalytic sets. The sequence

$$
0 \rightarrow \varnothing \mathscr{D} b_{\left[Z_{1} \cap Z_{2}\right]}^{*} \rightarrow \varnothing \mathscr{D} b_{\left[Z_{1}\right]}^{*} \oplus \varnothing \mathscr{D} b_{\left[Z_{2}\right]}^{*} \rightarrow \varnothing \mathscr{D} b_{\left[Z_{1} \cup Z_{2}\right]}^{*} \rightarrow 0
$$

is exact. 
Proof. - Since it is a local problem, we may assume that $X=\mathbb{R}^{2}$ and $Z_{i}$ is compact subanalytic. It suffices to show the exactness of the sequence:

$$
0 \rightarrow \& \mathscr{D} b_{\left[Z_{1} \cap Z_{2}\right]}^{*}(X) \rightarrow \varnothing \mathscr{D} b_{\left[Z_{1}\right]}^{*}(X) \oplus \phi \mathscr{D} b_{\left[Z_{2}\right]}^{*}(X) \rightarrow \& \mathscr{D} b_{\left[Z_{1} \cup Z_{2}\right]}^{*}(X) \rightarrow 0 .
$$

Then the injectivity is clear, and the surjectivity follows from Lemma 2.1.5. Using Lemma 3.1.3 instead of Lemma 3.1.2, we can prove the exactness of the middle by the same argument as in the proof of Theorem 3.1.4.

Note that, for the case $*=(s)$, the corollary can be also proved by taking the dual of (3.1) since all the vector spaces in (3.1) have FS topologies.

3.2. Stratified and tempered-stratified ultradistributions. - In this subsection, we assume that $X$ is a real analytic manifold with arbitrary dimension. For $U \subset X$ a subanalytic open set, we define the set of tempered-stratified ultradistributions as

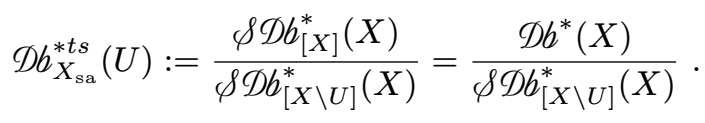

THEOREM 3.2.1. - Let $U$ be an open subanalytic subset of $X$.

1. The ring $\Gamma\left(\bar{U}, \mathscr{D}_{X}\right)$ acts on $\mathscr{D} b_{X_{\mathrm{sa}}}^{* t s}(U)$.

2. Let $V$ be an open subanalytic subset of $X$. Then we have the following exact sequence.

$$
\mathscr{D} b_{X_{\mathrm{sa}}}^{* t s}(U \cup V) \rightarrow \mathscr{D} b_{X_{\mathrm{sa}}}^{* t s}(U) \oplus \mathscr{D} b_{X_{\mathrm{sa}}}^{* t s}(V) \rightarrow \mathscr{D} b_{X_{\mathrm{sa}}}^{* t s}(U \cap V) \rightarrow 0 .
$$

Further, if $\operatorname{dim}_{\mathbb{R}}(X) \leq 2$, then the first morphism of the above sequence is injective. Hence, in this case, $\mathscr{D} b_{X_{\mathrm{sa}}}^{* t s}$ is a sheaf on $X_{\mathrm{sa}}$ and a $\varrho_{!} \mathscr{D}_{X}$ module.

3. If $X \backslash U$ is 1-regular, then $\mathscr{D} b_{X_{\mathrm{sa}}}^{* t s}(U)$ coincides with the sections of tempered ultradistributions of class $*$ on $U$, that is,

$$
\mathscr{D} b_{X_{\mathrm{sa}}}^{* t s}(U)=\mathscr{D} b_{X}^{* t}(U)
$$

Proof. - 1. Let $W \supset \bar{U}$ be an open subset in $X$ and $P \in \Gamma\left(W, \mathscr{D}_{X}\right)$. We choose a function $\varphi \in \mathscr{C}^{*}(X)$ with $\operatorname{supp} \varphi \subset W$ and $\varphi(x)=1$ in a neighborhood of $\bar{U}$. Then $\varphi P$ can be considered as a differential operator on $X$ with coefficients in $\mathscr{C}^{*}(X)$, and thus, it acts on $\mathscr{D} b_{X}^{* t}(U)$. This action does not depend on a choice of $\varphi$. Indeed, this follows from the fact that, for any $u \in \mathscr{D} b^{*}(X)$ and $\psi \in \mathscr{C}^{*}(X)$ with $\operatorname{supp} \psi \cap \bar{U}=\varnothing$, we have $\psi u \in \varnothing \mathscr{D} b_{[X \backslash U]}^{*}(X)$. 
2. The exactness is an immediate consequence of the following commutative diagram whose rows and columns are exact.

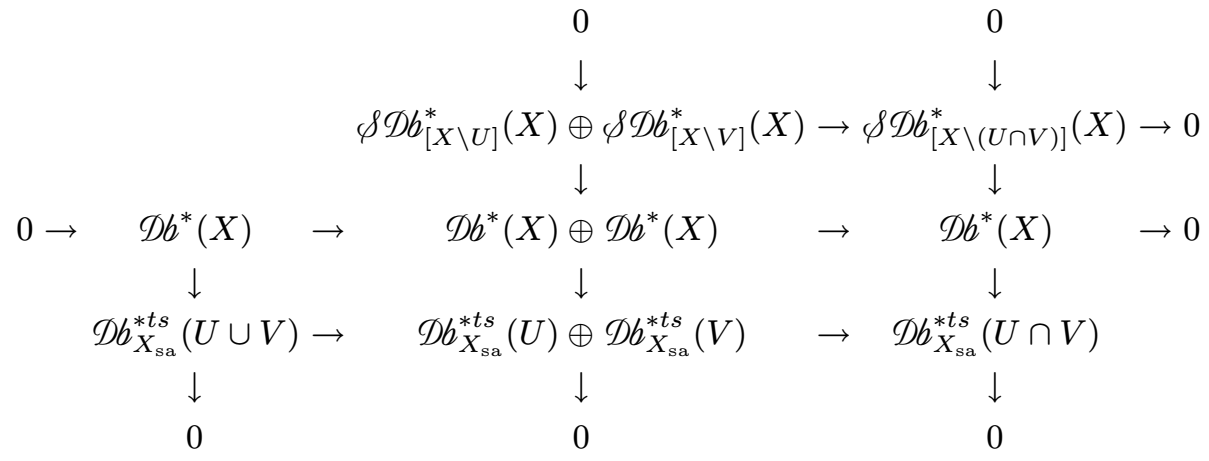

The assertion for the case $\operatorname{dim} X \leq 2$ comes from Corollary 3.1.5.

3. follows from Corollary 2.4.4.

3.3. Higher dimensional case. - Let $\mathscr{G}$ be a sheaf on $X_{\mathrm{sa}}$ and denote by $r_{V, U}$ the restriction map of $\mathscr{G}$ for $V \subset U$ open subanalytic subsets. Assume that $\mathscr{G}$ satisfies the following conditions.

1. If $U \in \mathrm{Op}\left(X_{\mathrm{sa}}\right)$ has smooth boundary, then $\mathscr{G}(U) \simeq \mathscr{D} b^{* t}(U)$. In particular, $\mathscr{G}(X)=\mathscr{D} b^{*}(X)$.

2. For any $U \in \mathrm{Op}\left(X_{\mathrm{sa}}\right), r_{U}:=r_{U, X}: \mathscr{D} b^{*}(X)=\mathscr{G}(X) \rightarrow \mathscr{G}(U)$ is surjective, i.e. $\mathscr{G}$ is quasi-injective.

Note that, since $\mathscr{G}$ is a sheaf in $X_{\text {sa }}$, for open subanalytic subsets $U$ and $V$, the sequence

$$
0 \rightarrow \mathscr{G}(U \cup V) \rightarrow \mathscr{G}(U) \oplus \mathscr{G}(V) \rightarrow \mathscr{G}(U \cap V)
$$

is exact.

If $\operatorname{dim} X=2$, the sheaf $\mathscr{D} b_{X_{\mathrm{sa}}}^{* t s}$ satisfies the the conditions 1. and 2. above. Let us prove that, if $\operatorname{dim} X>2$, then such a sheaf $\mathscr{G}$ on $X_{\text {sa }}$ does not exist. For $Z$ a subanalytic closed subset of $X$, set

$$
\mathcal{F}(Z):=\operatorname{ker}\left(\mathscr{D} b^{*}(X) \rightarrow \mathscr{G}(X \backslash Z)\right) \subset \mathscr{D} b^{*}(X) .
$$

Then, for any closed subanalytic sets $Z_{1} \subset Z_{2} \subset X$, there exists an injective morphism

$$
i_{Z_{2}, Z_{1}}: \mathcal{F}\left(Z_{1}\right) \hookrightarrow \mathcal{F}\left(Z_{2}\right)
$$

satisfying $i_{Z_{3}, Z_{1}}=i_{Z_{3}, Z_{2}} \circ i_{Z_{2}, Z_{1}}$. One checks easily that the sequence

$$
0 \rightarrow \mathcal{F}\left(Z_{1} \cap Z_{2}\right) \rightarrow \mathcal{F}\left(Z_{1}\right) \oplus \mathcal{F}\left(Z_{2}\right) \rightarrow \mathcal{F}\left(Z_{1} \cup Z_{2}\right) \rightarrow 0
$$

is exact. 
Using the fact that $i_{Z_{2}, Z_{1}}$ is injective, and $\mathcal{F}(Z) \subset \mathcal{F}(X \backslash B)=$ $\Gamma_{X \backslash B}\left(X, \mathscr{D} b^{*}\right)$, for any open ball $B$ with $Z \cap B=\varnothing$, it is easy to see that

$$
\mathcal{F}(Z) \subset \Gamma_{Z}\left(X, \mathscr{D} b^{*}\right)
$$

Further, if $Z$ is a smooth manifold we have

$$
\mathcal{F}(Z)=\Gamma_{Z}\left(X, \mathscr{D} b^{*}\right) \text {. }
$$

Now let $Z_{1}, Z_{2}$ be two smooth hypersurfaces of $X$, from the commutative diagram with exact rows

$$
\begin{aligned}
& 0 \rightarrow \mathcal{F}\left(Z_{1} \cap Z_{2}\right) \quad \rightarrow \quad \mathcal{F}\left(Z_{1}\right) \oplus \mathcal{F}\left(Z_{2}\right) \quad \rightarrow \quad \mathcal{F}\left(Z_{1} \cup Z_{2}\right)
\end{aligned}
$$

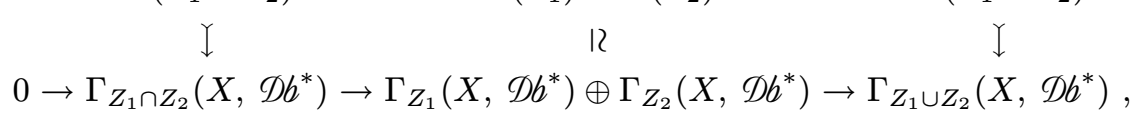

one obtains that

$$
\mathcal{F}\left(Z_{1} \cap Z_{2}\right)=\Gamma_{Z_{1} \cap Z_{2}}\left(X, \mathscr{D} b^{*}\right) .
$$

If $\operatorname{dim}(X)>2$, then we can find a pair of smooth hypersurfaces $Z_{1}$ and $Z_{2}$ such that $Z_{1} \cap Z_{2}$ consists of two smooth curves $W_{1}$ and $W_{2}$ tangentially intersecting at $p$. For example, let $X=\mathbb{R}^{3}$ with coordinates $(x, y, z), Z_{1}=$ $\{z=0\}$ and $Z_{2}=\left\{z+y^{2}-x^{2 m}=0\right\}$. Then, since $Z_{1} \cap Z_{2}=W_{1} \cup W_{2}$, we have the following exact sequence

$$
\begin{aligned}
\Gamma_{W_{1}}\left(X, \mathscr{D} b^{*}\right) \oplus \Gamma_{W_{2}}\left(X, \mathscr{D} b^{*}\right) & =\mathcal{F}\left(W_{1}\right) \oplus \mathcal{F}\left(W_{2}\right) \\
& \rightarrow \mathcal{F}\left(W_{1} \cup W_{2}\right)=\Gamma_{W_{1} \cup W_{2}}\left(X, \mathscr{D} b^{*}\right) \rightarrow 0 .
\end{aligned}
$$

This gives a contradiction. Indeed, the exactness of (3.3) implies that any ultradistributions supported on $W_{1} \cup W_{2}$ is the sum of ultradistributions supported in $W_{1}$ or $W_{2}$. When $W_{1}$ and $W_{2}$ are tangent at $p$, this is not true.

\section{Appendix A}

\section{Super growth indicators}

The aim of the appendix is to show Proposition A.7. To prove the proposition we need several lemmas and propositions. Their proofs are given only for the case $*=(s)$ in this paper as those for $*=\{s\}$ can be done by the similar technique.

Definition A.1. - We say that a $\mathscr{C}^{2}$-function $\varphi(t): \mathbb{R}_{\geq 0} \rightarrow \mathbb{R}$ is a super growth indicator if $\lim _{t \rightarrow \infty} \varphi^{\prime}(t)=\infty$ and $\varphi^{\prime \prime}(t) \geq 0$. Further, a function $\varphi(t)$ : $\mathbb{R}_{\geq 0} \rightarrow \mathbb{R}$ is said to be $a$ linear growth indicator if there exist a positive constant $h>0$ and a constant $C$ such that $\varphi(t)=h t+C$. 
Note that a super growth indicator is a convex function.

Let $\varphi_{1}(t), \varphi_{2}(t)$ be two super growth indicators, we write $\varphi_{1} \ll \varphi_{2}$ if and only if there exists $C \in \mathbb{R}$ such that, for any $t \in \mathbb{R}_{\geq 0}, \varphi_{1}(t) \leq \varphi_{2}(t)+C$.

LEMMA A.2. - 1. Let $\varphi(x)$ be a super growth indicator. There exists $\left\{\gamma_{k}\right\}_{k \in \mathbb{Z}_{>0}} \subset \mathbb{R}$ such that, for any $k \in \mathbb{Z}_{>0}$,

$$
\varphi(t) \geq k t+\gamma_{k} .
$$

2. For any $\left\{\gamma_{k}\right\}_{k \in \mathbb{Z}_{>0}} \subset \mathbb{R}$, there exists a super growth indicator $\varphi(t)$ such that

$$
\sup _{k \geq 1}\left(k t+\gamma_{k}\right) \geq \varphi(t)
$$

Proof. - Easy.

Let $X$ be a real analytic manifold and $A$ a closed subanalytic set, and let $A_{\alpha}$ be a stratum of a stratification $\left\{A_{\alpha}\right\}$ of $A$.

We set

$$
B\left(A_{\alpha}\right):=\underset{A_{\alpha} \subset \bar{A}_{\beta}}{\cup} \bar{A}_{\beta} .
$$

Clearly $B\left(A_{\alpha}\right)$ is a closed subanalytic subset of $X$.

Proposition A.3. - Let $X=\mathbb{R}^{n}$ and $A \subset X$ a closed subanalytic set with a 1-regular stratification $\left\{A_{\alpha}\right\}$. Assume $*=(s)$ (resp. $\left.*=\{s\}\right)$. Then for any $\alpha$ and any $F \in \& W_{A}^{*}(X)$ with $\left.F\right|_{\bar{A}_{\alpha}}=0$, there exists a super (resp. linear) growth indicator $\varphi_{\alpha}$ satisfying the following condition. For any $h>0$ (resp. some $h>0)$, there exists a constant $C_{h}$ such that, for any $\epsilon>0$,

$$
\|F\|_{B\left(A_{\alpha}, \epsilon\right), s, h} \leq C_{h} \exp \left(-\varphi_{\alpha}\left(\epsilon^{-\sigma}\right)\right),
$$

where $B\left(A_{\alpha}, \epsilon\right)=\left\{x \in B\left(A_{\alpha}\right) ; \operatorname{dist}\left(x, \bar{A}_{\alpha}\right) \leq \epsilon\right\}$ and $\sigma=\frac{1}{s-1}$.

Proof. - We may assume that $A=B\left(A_{\alpha}\right)$, in particular, the number of the strata is finite. Since the closure of each stratum $A_{\beta}$ is 1-regular, connected and compact, we can find a constant $\kappa_{\beta}$ such that for any $x, y \in \bar{A}_{\beta}$ there exists a subanalytic curve $l \subset \bar{A}_{\beta}$ joining $x$ and $y$ such that

$$
|l| \leq \kappa_{\beta}|x-y| \text {. }
$$

We set

$$
\kappa=\max \left\{\kappa_{\beta}\right\} .
$$

Let $y$ be a point in some stratum $A_{\beta}$. As $\bar{A}_{\alpha}$ is compact, there exists $x \in \bar{A}_{\alpha}$ such that

$$
\operatorname{dist}\left(y, \bar{A}_{\alpha}\right)=|y-x| \text {. }
$$

As $\bar{A}_{\alpha} \subset \bar{A}_{\beta}$, there exists a curve $l$ in $\bar{A}_{\beta}$ joining $x$ and $y$ such that

$$
|l| \leq \kappa|x-y| \text {. }
$$

TOME $139-2011-\mathrm{N}^{\mathrm{O}} 3$ 
Now, let $F=\left\{f_{\alpha}\right\}_{\alpha} \in \phi W_{A}^{(s)}(X)$ such that $\left.F\right|_{\bar{A}_{\alpha}}=0$. We have that, for any $x \in \bar{A}_{\alpha}, y \in \bar{A}_{\beta}, f_{\gamma}(y)=R_{m}\left(S_{\gamma} F ; y, x\right)$. Hence, from Lemma 2.2.5, it follows that

$$
\begin{aligned}
\left|f_{\gamma}(y)\right|=\left|R_{m}\left(S_{\gamma} F ; y, x\right)\right| & \leq \frac{(\sqrt{n}|l|)^{m+1}}{m !} \max _{|\eta|=|\gamma|+m+1} \sup _{y \in l}\left|f_{\eta}(y)\right| \\
& \leq \frac{(\sqrt{n} \kappa|x-y|)^{m+1}}{m !}(|\gamma|+m+1) !^{s} h^{|\gamma|+m+1}|| F \|_{A, s, h} \\
& \leq\left(2^{s+1} \sqrt{n} \kappa h|x-y|\right)^{m+1} m !^{s-1}(|\gamma|) !^{s}\left(2^{s} h\right)^{|\gamma|}\|F\|_{A, s, h} .
\end{aligned}
$$

Hence for any $h^{\prime}>0$ and $h>0$

$$
\frac{\left|f_{\gamma}(y)\right|}{|\gamma| !^{s}\left(2^{s} h^{\prime}\right)^{|\gamma|}} \leq\left(2^{s+1} \sqrt{n} \kappa h|x-y|\right)^{m+1} m !^{s-1}\left(\frac{h}{h^{\prime}}\right)^{|\gamma|}\|F\|_{A, s, h} .
$$

Since

$$
\inf _{m \in \mathbb{N}} t^{m+1} m !^{s-1} \leq C \exp \left(-B t^{-\sigma}\right)
$$

for some positive constants $B$ and $C$, we obtained

$$
\frac{\left|f_{\gamma}(y)\right|}{|\gamma| !^{s}\left(2^{s} h^{\prime}\right)^{|\gamma|}} \leq C\|F\|_{A, s, h}\left(\frac{h}{h^{\prime}}\right)^{|\gamma|} \exp \left(-B^{\prime}\left(\frac{1}{h}\right)^{\sigma}(|x-y|)^{-\sigma}\right)
$$

for a constant $B^{\prime}>0$.

By Lemma A.2, there exists a super growth indicator $\varphi$ such that

$$
\sup _{h>0} \frac{1}{C\|F\|_{A, s, h}} \exp \left(B^{\prime}\left(\frac{1}{h}\right)^{\sigma} t\right) \geq \exp (\varphi(t)) .
$$

Then, one checks easily that, for any $h^{\prime}>0$, there exists a constant $C_{h^{\prime}}>0$ such that:

$$
\sup _{h^{\prime}>h>0} \frac{1}{C\|F\|_{A, s, h}} \exp \left(B^{\prime}\left(\frac{1}{h}\right)^{\sigma} t\right) \geq \frac{1}{C_{h^{\prime}}} \exp (\varphi(t)) .
$$

Therefore, we obtain

$$
\begin{aligned}
\frac{\left|f_{\gamma}(y)\right|}{|\gamma| !^{s}\left(2^{s} h^{\prime}\right)^{|\gamma|}} & \leq \inf _{0<h<h^{\prime}} C\|F\|_{A, s, h}\left(\frac{h}{h^{\prime}}\right)^{|\gamma|} \exp \left(-B^{\prime}\left(\frac{1}{h}\right)^{\sigma}(|x-y|)^{-\sigma}\right) \\
& \leq \inf _{0<h<h^{\prime}} C\|F\|_{A, s, h} \exp \left(-B^{\prime}\left(\frac{1}{h}\right)^{\sigma}(|x-y|)^{-\sigma}\right) \\
& \leq C_{h^{\prime}} \exp \left(-\varphi\left(|x-y|^{-\sigma}\right)\right) .
\end{aligned}
$$

This entails the result.

Lemma A.4. - For any constant $C>1, \epsilon>0$ and any super growth indicator $\psi$, there exists a super growth indicator $\varphi$ satisfying the following conditions.

1. $\varphi(t) \ll \psi(t)$.

2. $\varphi^{\prime}(0)>0$ and $\varphi(0)=0$.

BULletin DE LA SOCIÉtÉ MATHÉmATIQUE DE FRANCE 
3. For any $s, t \in[1, \infty), \varphi(s t) \leq C s^{1+\epsilon} \varphi(t)$.

Proof. - Note that the third condition is equivalent to

$$
\varphi(s) \leq C\left(\frac{s}{t}\right)^{\epsilon} \frac{\varphi(t)}{t} s \quad s \geq t \geq 1
$$

Set

$$
\gamma=C^{\frac{1}{5}}>1
$$

Without loss of generality, we assume that $\psi(0)=0$ and $\psi^{\prime}(0)=M$, for some constant $M>1$. Let $\left\{\hat{x}_{k}\right\}_{k \in \mathbb{Z}_{>0}} \subset[0, \infty)$ satisfy:

1. $0=\hat{x}_{0}<\hat{x}_{1}<\hat{x}_{2}<\ldots$ and $\lim _{k \rightarrow \infty} \hat{x}_{k}=\infty$.

2. $\psi^{\prime}\left(\hat{x}_{k}\right)=M \gamma^{k}$.

Given an increasing sequence $\left\{x_{k}\right\}_{k \in \mathbb{Z} \geq 0} \subset \mathbb{R}$ with $x_{0}=0$ and $\lim _{k \rightarrow \infty} x_{k}=\infty$, we set

$$
g(x):=\sum_{i=0}^{k-1} \gamma^{i}\left(x_{i+1}-x_{i}\right)+\gamma^{k}\left(x-x_{k}\right) \quad x \in\left[x_{k}, x_{k+1}\right] .
$$

Let $\left\{x_{k}\right\}_{k \in \mathbb{Z} \geq 0}$ be an increasing sequence of real numbers satisfying the following conditions:

1. $x_{0}=0$ and $x_{1} \geq 2$,

2. $x_{k+1} \geq \max \left\{\gamma^{\frac{1}{\epsilon}} x_{k}, \hat{x}_{k+1}\right\}$ for $k \in \mathbb{N}$,

3. $g\left(x_{k+1}\right) \geq \gamma^{k-1} x_{k+1}$ for $k \in \mathbb{N}$.

Note that such a sequence $\left\{x_{k}\right\}_{k}$ always exists as, for fixed $k$, we have

$$
\lim _{x \rightarrow \infty} \frac{\sum_{i=0}^{k-1} \gamma^{i}\left(x_{i+1}-x_{i}\right)+\gamma^{k}\left(x-x_{k}\right)}{x}=\gamma^{k} .
$$

Clearly, if $t \leq s$, then $\left(\frac{s}{t}\right)^{\epsilon} \geq 1>\gamma^{-2}$. More precisely, for $0<t \leq s$ with $t \in\left[x_{l}, x_{l+1}\right]$ and $s \in\left[x_{k}, x_{k+1}\right](l<k)$, we easily obtain

$$
\left(\frac{s}{t}\right)^{\epsilon} \geq\left(\frac{x_{k}}{x_{l+1}}\right)^{\epsilon} \geq \gamma^{k-l-2}
$$

Moreover, since the function $g(t)$ is convex with $g(0)=0$, the function $\frac{g(t)}{t}$ is an increasing function of $t$. Hence, for any $t \in\left[x_{l}, x_{l+1}\right](1 \leq l)$, we have

$$
\frac{g(t)}{t} \geq \frac{g\left(x_{l}\right)}{x_{l}} \geq \gamma^{l-2}
$$

It follows that, for $0<t \leq s, s \in\left[x_{k}, x_{k+1}\right]$,

$$
\gamma^{k}=\gamma^{4} \gamma^{k-l-2} \gamma^{l-2} \leq \frac{1}{\gamma} C\left(\frac{s}{t}\right)^{\epsilon} \frac{g(t)}{t},
$$

TOME $139-2011-\mathrm{N}^{\mathrm{O}} 3$ 
and

$$
g(s)=\sum_{i=0}^{k-1} \gamma^{i}\left(x_{i+1}-x_{i}\right)+\gamma^{k}\left(s-x_{k}\right) \leq \gamma^{k} s \leq \frac{1}{\gamma} C\left(\frac{s}{t}\right)^{\epsilon} \frac{g(t)}{t} s .
$$

Since $\psi(s)$ is an increasing convex function, we have

$$
\begin{aligned}
g(s) & =\sum_{i=0}^{k-1} \gamma^{i}\left(x_{i+1}-x_{i}\right)+\gamma^{k}\left(s-x_{k}\right) \\
& =\sum_{i=0}^{k-1} \frac{\psi^{\prime}\left(\hat{x}_{i}\right)}{M}\left(x_{i+1}-x_{i}\right)+\frac{\psi^{\prime}\left(\hat{x}_{k}\right)}{M}\left(s-x_{k}\right) \\
& \leq \frac{1}{M}\left(\sum_{i=0}^{k-1} \psi^{\prime}\left(x_{i}\right)\left(x_{i+1}-x_{i}\right)+\psi^{\prime}\left(x_{k}\right)\left(s-x_{k}\right)\right) \\
& \leq \frac{1}{M} \psi(s) .
\end{aligned}
$$

Hence, the continuous convex increasing function $g(s)$ satisfies:

$$
g(s t) \leq \frac{1}{\gamma} C s^{1+\epsilon} g(t) \quad \text { for } s \in[1, \infty) \text { and } t \in[0, \infty)
$$

and

$$
g(t) \leq \frac{1}{M} \psi(t)
$$

Let $\chi_{h}(x)$ be a non-negative $\mathscr{C}^{\infty}(\mathbb{R})$ function such that

$$
\operatorname{supp}\left(\chi_{h}(x)\right) \subset\{|x| \leq h\} \quad \text { and } \int_{\mathbb{R}} \chi_{h}(x) d x=1 .
$$

For $0<h<1$, set

$$
\varphi_{h}(t)=\left\{\begin{array}{lr}
t & 0 \leq t \leq 1 \\
\int_{\mathbb{R}} g(t-x) \chi_{h}(x) d x & t \geq 1
\end{array}\right.
$$

Then we have $\varphi_{h} \in \mathscr{C}^{\infty}(\mathbb{R})$ and

$$
g(t) \leq \varphi_{h}(t) \leq g(t+h)
$$

Hence, we obtain

$$
\varphi_{h}(s t-h) \leq g(s t) \leq \frac{1}{\gamma} C s^{1+\epsilon} g(t) \leq \frac{1}{\gamma} C s^{1+\epsilon} \varphi_{h}(t) .
$$

Now, we replace $s$ by $(1+h) s$ into (A.3). Then we get, for sufficiently small $h>0$ and $s, t \in[1, \infty)$,

$$
\varphi_{h}(s t) \leq \varphi_{h}((1+h) s t-h) \leq \frac{(1+h)^{1+\epsilon}}{\gamma} C s^{1+\epsilon} \varphi_{h}(t) \leq C s^{1+\epsilon} \varphi_{h}(t) .
$$


Further, it follows from $g(t) \leq \frac{1}{M} \psi(t)$ that for sufficiently small $h>0$ and $t \geq 1$ we have

$$
\varphi_{h}(t) \leq \psi(t)
$$

The conclusion follows.

LEMmA A.5. - Let $\sigma>0$. For any super growth indicator $\varphi(t)$, there exist a holomorphic function $p(\xi)$ on $\mathbb{C} \backslash \mathbb{R}_{\leq 0}$ and a super growth indicator $\varrho(t)$ satisfying the following conditions.

1. There exists $C>0$ such that, for any $\xi \in \mathbb{C} \backslash \mathbb{R}_{\leq 0}$,

$$
|p(\xi)| \leq C \exp \left(\varphi\left(|\xi|^{\sigma}\right)\right)
$$

holds. Moreover $p(\xi)$ is real valued for $\xi \in \mathbb{R}_{>0}$.

2. The inequality

$$
\exp \left(\varrho\left(|\xi|^{\sigma}\right)\right) \leq|p(\xi)|
$$

holds for any $\xi \in\left\{\xi=r e^{i \vartheta} \in \mathbb{C} ; r>0,|\vartheta|<\min \left\{\frac{\pi}{4}, \frac{\pi}{8 \sigma}\right\}\right\}$.

Proof. - It is enough to construct an entire function $p(\xi)$ for $\sigma=\frac{1}{2}$ that satisfies the estimate (A.4) for any $\xi \in \mathbb{C}$ and (A.5) on $\left\{r e^{i \vartheta} \in \mathbb{C} ; r>0,|\vartheta|<\frac{\pi}{4}\right\}$. Then, for an arbitrary $\sigma>0$, the holomorphic function $p\left(\xi^{2 \sigma}\right)$ on $\mathbb{C} \backslash \mathbb{R}_{\leq 0}$ gives a required one.

We suppose $\sigma=\frac{1}{2}$ in what follows. Let $\psi(t)$ be a super growth indicator satisfying the conditions 2. and 3. in Lemma A.4 for some $\epsilon>1$ and $C>0$ which will be determined later on.

For $s \geq 0$, we set

$$
g(s):=\sigma s \psi^{\prime}(s)
$$

As

$$
g^{\prime}(s)=\sigma\left(\psi^{\prime}(s)+s \psi^{\prime \prime}(s)\right) \geq \sigma \psi^{\prime}(0)>0,
$$

we have that $g(s)$ is a strictly increasing function and $\lim _{s \rightarrow \infty} g(s)=\infty$.

Now, let $\left\{\xi_{k}\right\}$ be a sequence such that

$$
k=g\left(\xi_{k}^{\sigma}\right) \quad k=1,2, \ldots
$$

holds for any $k \in \mathbb{Z}_{>0}$. Then, since $g(s)$ is strictly increasing, we have

$$
\xi_{1}<\xi_{2}<\ldots, \quad \text { and } \lim _{k \rightarrow \infty} \xi_{k}=\infty .
$$

For any $t>0$, we set

$$
\begin{aligned}
n(t) & :=\left\{\text { the number of } \xi_{k} \text { such that }\left|\xi_{k}\right| \leq t\right\} \\
N(t) & :=\int_{0}^{t} \frac{n(\lambda)}{\lambda} d \lambda .
\end{aligned}
$$

TOME $139-2011-\mathrm{N}^{\circ} 3$ 
Moreover, for any $\xi_{k} \leq s<\xi_{k+1}$ we have

$$
n(s)=n\left(\xi_{k}\right)=k=g\left(\xi_{k}^{\sigma}\right) \leq g\left(s^{\sigma}\right),
$$

hence

$$
\begin{aligned}
N(s) & \leq \int_{0}^{s} \frac{n(t)}{t} d t \leq \int_{0}^{s} \frac{g\left(t^{\sigma}\right)}{t} d t \\
& =\int_{0}^{s} \frac{d}{d t} \psi\left(t^{\sigma}\right) d t=\psi\left(s^{\sigma}\right)-\psi(0)=\psi\left(s^{\sigma}\right) .
\end{aligned}
$$

Set

$$
p(\xi):=\Pi_{k \geq 1}\left(1+\frac{\xi}{\xi_{k}}\right) .
$$

By the Lindelöf Theorem (see proof of Proposition 4.6, page 59 of [8]) we conclude that $p(\xi)$ is absolutely convergent in $\mathbb{C}$. The same theorem give the estimate

$$
\log \sup _{|\xi|=t}|p(\xi)| \leq \int_{0}^{\infty} \frac{t N(\lambda)}{(t+\lambda)^{2}} d \lambda .
$$

Thus we get

$$
\begin{aligned}
\log \sup _{|\xi|=t}|p(\xi)| & \leq \int_{0}^{\infty} \frac{t \psi\left(\lambda^{\sigma}\right)}{(t+\lambda)^{2}} d \lambda \\
& =\int_{0}^{t} \frac{t \psi\left(\lambda^{\sigma}\right)}{(t+\lambda)^{2}} d \lambda+\int_{t}^{\infty} \frac{t \psi\left(\lambda^{\sigma}\right)}{(t+\lambda)^{2}} d \lambda
\end{aligned}
$$

The first term of right hand side of (A.6) satisfies

$$
\int_{0}^{t} \frac{t \psi\left(\lambda^{\sigma}\right)}{(t+\lambda)^{2}} d \lambda \leq \frac{1}{t} \int_{0}^{t} \psi\left(\lambda^{\sigma}\right) d \lambda \leq \psi\left(t^{\sigma}\right) .
$$

We estimate the second term of the right hand side of (A.6) as follows. Let $\psi$ be a super growth indicator satisfying the condition 3. in Lemma A.4 for $C=2$ and $\epsilon=\frac{1}{\sqrt{\sigma}}-1$. Then we have

$$
\begin{aligned}
\int_{t}^{\infty} \frac{t \psi\left(\lambda^{\sigma}\right)}{(t+\lambda)^{2}} d \lambda & =\int_{1}^{\infty} \frac{\psi\left(t^{\sigma} \lambda^{\sigma}\right)}{(1+\lambda)^{2}} d \lambda \\
& \leq 2 \int_{1}^{\infty} \frac{\lambda^{\sigma(1+\epsilon)} \psi\left(t^{\sigma}\right)}{1+\lambda^{2}} d \lambda \leq 2\left(\int_{1}^{\infty} \frac{1}{\lambda^{2-\sqrt{\sigma}}} d \lambda\right) \psi\left(t^{\sigma}\right) .
\end{aligned}
$$

Hence we obtain

$$
\log \sup _{|\xi|=t}|p(\xi)| \leq C_{\sigma} \psi\left(t^{\sigma}\right)
$$

where $C_{\sigma}$ depends only on $\sigma$. Therefore $p(\xi)$ satisfies the condition 1 . of Lemma A.5 when $\psi(t)$ is a super growth indicator given by Lemma A.4 with the indicator $\frac{\varphi(t)}{C_{\sigma}}$ and the constants $C=2$ and $\epsilon=\frac{1}{\sqrt{\sigma}}-1$. 
Now, for $k \in \mathbb{Z}_{>0}$, set

$$
l_{k}:=\frac{k^{\frac{1}{\sigma}}}{\xi_{k}} .
$$

Let us prove that, for $k \rightarrow \infty, l_{k} \rightarrow \infty$. Indeed, unless $l_{k} \rightarrow \infty$, then there exists $M>0$ and an increasing sequence of natural numbers $\left\{k_{p}\right\}$ with $l_{k_{p}} \leq M$. Since

$$
k_{p}=g\left(\xi_{k_{p}}^{\sigma}\right)=\sigma \xi_{k_{p}}^{\sigma} \psi^{\prime}\left(\xi_{k_{p}}^{\sigma}\right)=\sigma \frac{k_{p}}{l_{k_{p}}^{\sigma}} \psi^{\prime}\left(\frac{k_{p}}{l_{k_{p}}^{\sigma}}\right)
$$

holds, we have

$$
l_{k_{p}}^{\sigma}=\sigma \psi^{\prime}\left(\frac{k_{p}}{l_{k_{p}}^{\sigma}}\right) .
$$

Since $\psi^{\prime}(t) \rightarrow \infty(t \rightarrow \infty)$ and $\frac{k_{p}}{l_{k_{p}}^{\sigma}} \rightarrow \infty(p \rightarrow \infty)$, the right hand side of (A.7) tends to $\infty$. This contradicts to the fact that the left hand side of (A.7) is bounded.

Now, $p(\xi)$ can be written in the form:

$$
p(\xi)=\Pi_{k \geq 1}\left(1+\frac{l_{k} \xi}{k^{\frac{1}{\sigma}}}\right) .
$$

If $\xi \in D=\{|\Im \xi| \leq \Re \xi\}$, we have

$$
\left|1+\frac{l_{k} \xi}{k^{\frac{1}{\sigma}}}\right| \geq 1+\frac{l_{k} \Re \xi}{k^{\frac{1}{\sigma}}} \geq 1+\frac{l_{k}|\xi|}{\sqrt{2} k^{\frac{1}{\sigma}}} .
$$

Since $l_{k} \rightarrow \infty$, for any given $L>0$ there exists $k_{0}$ such that $l_{k} \geq \sqrt{2} L\left(k \geq k_{0}\right)$. Hence, for $\xi \in D$, we get

$$
|p(\xi)| \geq \frac{\left|\Pi_{k \leq k_{0}}\left(1+\frac{l_{k} \xi}{k^{\frac{1}{\sigma}}}\right)\right|}{\Pi_{k \leq k_{0}}\left(1+\frac{L|\xi|}{k^{\frac{1}{\sigma}}}\right)} \Pi_{1 \leq k}\left(1+\frac{L|\xi|}{k^{\frac{1}{\sigma}}}\right) \geq C_{L} \Pi_{1 \leq k}\left(1+\frac{L|\xi|}{k^{\frac{1}{\sigma}}}\right)
$$

for some $C_{L}>0$. It is well known that there exist $A, B>0$ such that, for $t \geq 0$,

$$
\Pi_{1 \leq k}\left(1+\frac{t}{k^{\frac{1}{\sigma}}}\right) \geq A \exp \left(B t^{\sigma}\right) .
$$

Therefore we have that for any $L>0$

$$
|p(\xi)| \geq A C_{L} \exp \left(B L^{\sigma}|\xi|^{\sigma}\right)=\exp \left(B L^{\sigma}|\xi|^{\sigma}+\log \left(A C_{L}\right)\right) \quad \xi \in D .
$$

This implies that there exists a super growth indicator $\varrho(t)$ satisfying

$$
|p(\xi)| \geq \exp \varrho\left(|\xi|^{\sigma}\right) \quad \xi \in D
$$


Lemma A.6. - Let $s>1$. Assume $*=(s)$ (resp. $*=\{s\})$. Then for any compact set $K \subset \mathbb{R}^{n}$ and for any super (resp. any linear) growth indicator $\varphi$, there exists a family of functions $\left\{\chi_{\epsilon}(x)\right\}_{\epsilon>0} \subset \mathscr{C}^{*}\left(\mathbb{R}^{n}\right)$ satisfying the following conditions.

1. For any $h>0$ (resp. some $h>0$ ) there exists a constant $C_{h}$ such that

$$
\left\|\chi_{\epsilon}(x)\right\|_{\mathbb{R}^{n}, s, h} \leq C_{h} \exp \left(\varphi\left(\epsilon^{-\sigma}\right)\right) \quad \text { for any } \epsilon>0 \text {. }
$$

2. $\operatorname{supp}\left(\chi_{\epsilon}(x)\right) \subset K_{\epsilon}$.

3. $\chi_{\epsilon}(x) \geq 0$ and, if $x \in K_{\frac{\epsilon}{2}}, \chi_{\epsilon}(x)=1$.

Where $K_{\epsilon}$ denotes the set $\{x \in X ; \operatorname{dist}(x, K) \leq \epsilon\}$ and $\sigma=\frac{1}{s-1}$.

Proof. - Let $\psi$ be a super growth indicator, $p(\xi)$ a holomorphic function as given in Lemma A.5 with the super growth indicator $\psi(t)$ and $\sigma>0$. We set

$$
f(x)=\left\{\begin{array}{cc}
\frac{1}{p\left(x^{-1}\right)} & x>0, \\
0 & x \leq 0 .
\end{array}\right.
$$

One checks easily that $f(z)$ is holomorphic in the sector

$$
S=\{z \in \mathbb{C} ;|\arg (z)|<\kappa\},
$$

for some $\kappa>0$, and that there exists a super growth indicator $\varrho$ such that

$$
|f(z)|<\exp \left(-\varrho\left(|z|^{-\sigma}\right)\right)
$$

holds for $z \in S$. Hence, using the Cauchy inequality, it is easy to see that $f(x) \in \mathscr{C}^{(s)}(\mathbb{R})$, that is, for $h>0$ and any compact set $K \subset \mathbb{R}$ we have

$$
\|f(x)\|_{K, s, h}<\infty \text {. }
$$

Set

$$
g_{\epsilon}(x):=f(x+2 \epsilon) f(-x+2 \epsilon) .
$$

Then, for $0<\epsilon<1$, we have

$$
\left\|g_{\epsilon}(x)\right\|_{\mathbb{R}, s, h} \leq\|f(x)\|_{[0,4], s, \frac{h}{2}}^{2}
$$

and $\operatorname{supp}\left(g_{\epsilon}(x)\right) \subset\{|x| \leq 2 \epsilon\}$. Moreover, there exists $M>0$ such that, for any $x>0$,

$$
|f(x)| \geq M \exp \left(-\psi\left(|x|^{-\sigma}\right)\right)
$$

Hence, we get

$$
g_{\epsilon}(x) \geq M \exp \left(-2 \psi\left(\epsilon^{-\sigma}\right)\right)
$$

for $x \in[-\epsilon, \epsilon]$. Thus

$$
\int_{\mathbb{R}} g_{\epsilon}(x) d x \geq 2 \epsilon M \exp \left(-2 \psi\left(\epsilon^{-\sigma}\right)\right) .
$$


Now, set

Then $\hat{g}_{\epsilon}$ satisfies

$$
\hat{g}_{\epsilon}(x):=\frac{g_{\epsilon}(x)}{\int_{\mathbb{R}} g_{\epsilon}(x) d x} .
$$

$$
\left\|\hat{g}_{\epsilon}\right\|_{\mathbb{R}, s, h} \leq\|f\|_{[0,4], s, \frac{h}{2}}^{2} \frac{1}{2 \epsilon M} \exp \left(2 \psi\left(\epsilon^{-\sigma}\right)\right) \leq M_{h} \exp \left(3 \psi\left(\epsilon^{-\sigma}\right)\right)
$$

for some constant $M_{h}$ that depends only on $h$. Set

$$
\hat{g}_{\epsilon}\left(x_{1}, \ldots, x_{n}\right):=\hat{g}_{\epsilon}\left(x_{1}\right) \hat{g}_{\epsilon}\left(x_{2}\right) \ldots \hat{g}_{\epsilon}\left(x_{n}\right) .
$$

Then $\hat{g}_{\epsilon}(x)$ satisfies the followings conditions.

1. For any $h>0$ there exists $M_{h}>0$ such that

$$
\left\|\hat{g}_{\epsilon}\left(x_{1}, \ldots, x_{n}\right)\right\|_{\mathbb{R}^{n}, s, h} \leq M_{h} \exp \left(3 n \psi\left(\epsilon^{-\sigma}\right)\right) \quad \text { for any } \epsilon>0 .
$$

2.

$$
\int_{\mathbb{R}^{n}} \hat{g}_{\epsilon}(x) d x=1
$$

3.

$$
\operatorname{supp}\left(\hat{g}_{\epsilon}(x)\right) \subset\left\{x \in \mathbb{R}^{n} ;|x| \leq 2 \sqrt{n} \epsilon\right\} .
$$

Let $\hat{\chi}_{K_{\frac{3 \epsilon}{4}}}$ denote the characteristic function of $K_{\frac{3 \epsilon}{4}}$. If we chose

$$
\psi(t)=\frac{1}{3 n} \varphi\left((8 \sqrt{n})^{-\sigma} t\right)
$$

then

$$
\chi_{\epsilon}:=\hat{g}_{\frac{\epsilon}{8 \sqrt{n}}} * \hat{\chi}_{K_{\frac{3 \epsilon}{4}}}
$$

satisfies the required conditions.

Proposition A.7. - Let $X$ be a real analytic manifold and $A \subset X$ a compact subanalytic set. Then $W_{A}^{*}(X)$ is a dense subset of the locally convex topological vector space $\& W_{A}^{*}(X)$.

Proof. - Take a 1-regular stratification $\left\{A_{\alpha}\right\}$ of $A$ and fix it. Let $A_{\alpha}$ be a stratum. Set

$$
Z\left(A_{\alpha}\right):=\bigcup_{\bar{A}_{\alpha} \cap \bar{A}_{\beta} \neq \varnothing} \bar{A}_{\beta}
$$

and

$$
U\left(A_{\alpha}\right):=\bigcup_{A_{\alpha} \subset \bar{A}_{\beta}} A_{\beta} .
$$

Then $Z\left(A_{\alpha}\right)$ is compact and it is a closed neighborhood of $\bar{A}_{\alpha}$ in $A$. Hence by the partition of unity, we may assume that $X=\mathbb{R}^{n}$ and $A$ is a compact subanalytic set. 
Let $\Lambda_{k}\left(\right.$ resp. $\left.\Lambda_{\leq k}\right)$ be the set of indices $\alpha \in \Lambda$ such that $\operatorname{dim}\left(A_{\alpha}\right)=k$ (resp. $\left.\operatorname{dim}\left(A_{\alpha}\right) \leq k\right)$. For $\epsilon>0$, let us determine a family of closed sets $\left\{W_{\epsilon, \alpha}\right\}_{\alpha \in \Lambda}$ in $X$ and a family of positive constants $\left\{l_{\epsilon, \alpha}\right\}_{\alpha \in \Lambda}$ in the following way.

If $k=0$, then for any $\alpha \in \Lambda_{0}$,

1. $W_{\epsilon, \alpha}=A_{\alpha}$,

2. let $l_{\epsilon, \alpha}>0$ be such that $\epsilon>l_{\epsilon, \alpha}$ and $\left(W_{\epsilon, \alpha, l_{\epsilon, \alpha}} \cap A\right) \subset U\left(A_{\alpha}\right)$. Here $W_{\epsilon, \alpha, l_{\epsilon, \alpha}}=\left\{x \in X ; \operatorname{dist}\left(x, W_{\epsilon, \alpha}\right) \leq l_{\epsilon, \alpha}\right\}$.

Suppose that we already determined $W_{\epsilon, \alpha}$ and $l_{\epsilon, \alpha}$ for every $\alpha \in \Lambda_{\leq k-1}$. Set

$$
\epsilon_{k-1}=\min _{\alpha \in \Lambda_{\leq k-1}} l_{\epsilon, \alpha} .
$$

First, let $\left\{W_{\epsilon, \alpha}\right\}_{\alpha \in \Lambda_{k}}$ be such that

$$
\bar{A}_{\alpha} \backslash\left(\bigcup_{A_{\beta} \subset \bar{A}_{\alpha}, \beta \in \Lambda_{\leq k-1}} W_{\epsilon, \beta, \frac{1}{3} l_{\epsilon, \beta}}\right) \subset W_{\epsilon, \alpha} \subset A_{\alpha} .
$$

Note that the set

$$
\bigcup_{A_{\beta} \subset \bar{A}_{\alpha}, \beta \in \Lambda_{\leq k-1}} W_{\epsilon, \beta, \frac{1}{3} l_{\epsilon, \beta}}
$$

is a neighborhood of $\bar{A}_{\alpha} \backslash A_{\alpha}$. Since $W_{\epsilon, \alpha} \cap W_{\epsilon, \beta}=\varnothing$ for any $\alpha \neq \beta \in \Lambda_{k}$, there exists a constant $\delta_{\epsilon, k}>0$

$$
\delta_{\epsilon, k}=\frac{1}{3} \min _{\alpha \neq \beta \in \Lambda_{k}} \operatorname{dist}\left(W_{\epsilon, \alpha}, W_{\epsilon, \beta}\right) .
$$

Then, for $\alpha \in \Lambda_{k}$, let $l_{\epsilon, \alpha}$ satisfy:

1. $l_{\epsilon, \alpha}<\min \left\{\delta_{\epsilon, k}, \epsilon_{k-1}\right\}$,

2. $\left(W_{\epsilon, \alpha, l_{\epsilon, \alpha}} \cap A\right) \subset U\left(A_{\alpha}\right)$,

3. for any $\beta \in \Lambda_{\leq k-1}$ with $A_{\beta} \cap \bar{A}_{\alpha}=\varnothing, W_{\epsilon, \alpha, l_{\epsilon, \alpha}} \cap W_{\epsilon, \beta, l_{\epsilon, \beta}}=\varnothing$ holds.

Note that such an $l_{\epsilon, \alpha}$ always exists. Indeed, $W_{\epsilon, \alpha} \subset A_{\alpha}$ is a compact set and $U\left(A_{\alpha}\right)$ is an open subset in $A$. Hence, there exists $l_{\epsilon, \beta}$ satisfying Condition 2. Since $A_{\beta} \cap \bar{A}_{\alpha}=\varnothing$ implies $U\left(A_{\beta}\right) \cap A_{\alpha}=\varnothing$, we have by the induction hypothesis

$$
W_{\epsilon, \beta, l_{\epsilon, \beta}} \cap W_{\epsilon, \alpha}=W_{\epsilon, \beta, l_{\epsilon, \beta}} \cap A \cap W_{\epsilon, \alpha} \subset U\left(A_{\beta}\right) \cap A_{\alpha}=\varnothing .
$$

As $W_{\epsilon, \beta, l_{\epsilon, \beta}}$ and $W_{\epsilon, \alpha}$ are closed sets, the condition 3 . can be fullfilled.

Let $\varphi$ be a super growth indicator. By Lemma A.6, for any $h>0$, there exist $C_{h}>0$ and a family $\left\{\chi_{\epsilon, \beta}\right\} \subset \mathscr{C}^{(s)}(X)$ such any for any $\epsilon, \beta$

(a) $\chi_{\epsilon, \beta}(x)=1$ for $x \in W_{\epsilon, \beta, \frac{1}{2} l_{\epsilon, \beta}}$,

(b) $\operatorname{supp} \chi_{\epsilon, \beta}(x) \subset W_{\epsilon, \beta, l_{\epsilon, \beta}}$,

(c) $\left\|\chi_{\epsilon, \beta}\right\|_{\mathbb{R}^{n}, s, h} \leq C_{h} \exp \left(\varphi\left(l_{\epsilon, \beta}^{-\sigma}\right)\right)$. 
Since $\cup_{\beta} W_{\epsilon, \beta, \frac{1}{2} l_{\epsilon, \beta}}$ is a neighborhood of $A$, we have

$$
\prod_{\beta \in \Lambda}\left(1-\chi_{\epsilon, \beta}(x)\right)=0
$$

in a neighborhood of $A$.

Now, let us prove that, for any indices $\beta_{1}$ and $\beta_{2}$ with $\beta_{1} \npreceq \beta_{2}$ and $\beta_{2} \npreceq \beta_{1}$, we have

$$
\chi_{\epsilon, \beta_{1}}(x) \chi_{\epsilon, \beta_{2}}(x) \equiv 0
$$

Here $\beta_{1} \preceq \beta_{2}$ means $A_{\beta_{1}} \subset \bar{A}_{\beta_{2}}$. Indeed, if $\operatorname{dim} A_{\beta_{1}}=\operatorname{dim} A_{\beta_{2}}$ and $\beta_{1} \neq \beta_{2}$, then if follows from the condition 1 of $l_{\epsilon, \beta}$ and $l_{\epsilon, \beta_{i}}<\frac{1}{3} \operatorname{dist}\left(W_{\epsilon, \beta_{1}}, W_{\epsilon, \beta_{2}}\right)$ $(i=1,2)$ that we have

$$
\operatorname{supp}\left(\chi_{\epsilon, \beta_{1}}\right) \cap \operatorname{supp}\left(\chi_{\epsilon, \beta_{2}}\right) \subset W_{\epsilon, \beta_{1}, l_{\epsilon, \beta_{1}}} \cap W_{\epsilon, \beta_{2}, l_{\epsilon, \beta_{2}}}=\varnothing .
$$

Therefore we may assume $\operatorname{dim} A_{\beta_{1}}<\operatorname{dim} A_{\beta_{2}}$. Since $\beta_{1} \npreceq \beta_{2}$ implies $A_{\beta_{1}} \cap$ $\bar{A}_{\beta_{2}}=\varnothing$, the relations

$$
\operatorname{supp}\left(\chi_{\epsilon, \beta_{1}}\right) \cap \operatorname{supp}\left(\chi_{\epsilon, \beta_{2}}\right) \subset W_{\epsilon, \beta_{1}, l_{\epsilon, \beta_{1}}} \cap W_{\epsilon, \beta_{2}, l_{\epsilon, \beta_{2}}}=\varnothing
$$

follow from the condition 3 .

Then, from (A.8) and (A.9), we obtain that

$$
1=\sum_{i=1}^{\# \Lambda} \sum_{\beta_{1} \prec \beta_{2} \prec \cdots \prec \beta_{i} \in \Lambda}(-1)^{i} \chi_{\epsilon, \beta_{1}}(x) \chi_{\epsilon, \beta_{2}}(x) \ldots \chi_{\epsilon, \beta_{i}}(x)
$$

in a neighborhood of $A$. Here $\beta_{1} \prec \beta_{2}$ implies that $\beta_{1} \neq \beta_{2}$ and $A_{\beta_{1}} \subset \bar{A}_{\beta_{2}}$.

Let $F \in \phi W_{A}^{*}(X)$. Since we have $\phi W_{A}^{*}(X)=\varnothing W_{\left\{A_{\alpha}\right\}}^{*}(X)$, then, for any $\alpha$ there exists $g_{\alpha}(x) \in \mathscr{C}^{(s)}(X)$ such that $j_{\bar{A}_{\alpha}}\left(g_{\alpha}\right)=\left.F\right|_{\bar{A}_{\alpha}}$. Then, by Proposition A.3, there exists a super growth indicator $\psi(t)$ such that, for any $h>0$ and any $\alpha$, there exists a constant $C_{h}^{\prime}$ such that, for any $l>0$,

$$
\left\|j_{A}\left(g_{\alpha}\right)-F\right\|_{B\left(A_{\alpha}, l\right), s, h} \leq C_{h}^{\prime} \exp \left(-\psi\left(l^{-\sigma}\right)\right) .
$$

Set

$$
g_{\epsilon}(x):=\sum_{i=1}^{\# \Lambda} \sum_{\beta_{1} \prec \beta_{2} \prec \cdots \prec \beta_{i} \in \Lambda}(-1)^{i} \chi_{\epsilon, \beta_{1}}(x) \chi_{\epsilon, \beta_{2}}(x) \ldots \chi_{\epsilon, \beta_{i}}(x) g_{\beta_{i}}(x) \in \mathscr{C}^{(s)}(X) \text {. }
$$

We are going to show that $j_{A}\left(g_{\epsilon}\right)$ converges to $F$ with respect to the topology of $\delta W_{A}^{*}(X)$ when $\epsilon \rightarrow 0$. This will complete the proof. 
Let us fix a stratum $A_{\alpha}$. We have

$$
\begin{aligned}
& j_{\bar{A}_{\alpha}}\left(g_{\epsilon}\right)-\left.F\right|_{\bar{A}_{\alpha}}= \\
& =j_{\bar{A}_{\alpha}}\left(g_{\epsilon}\right)-\left.\sum_{i=1}^{\# \Lambda} \sum_{\beta_{1} \prec \beta_{2} \prec \cdots \prec \beta_{i} \in \Lambda} j_{\bar{A}_{\alpha}}\left((-1)^{i} \chi_{\epsilon, \beta_{1}}(x) \chi_{\epsilon, \beta_{2}}(x) \ldots \chi_{\epsilon, \beta_{i}}(x)\right) F\right|_{\bar{A}_{\alpha}} \\
& =\left.\sum_{i=1}^{\# \Lambda} \sum_{\beta_{1} \prec \beta_{2} \prec \cdots \prec \beta_{i} \in \Lambda} j_{\bar{A}_{\alpha}}\left((-1)^{i} \chi_{\epsilon, \beta_{1}}(x) \chi_{\epsilon, \beta_{2}}(x) \ldots \chi_{\epsilon, \beta_{i}}(x)\right)\left(j_{A}\left(g_{\beta_{i}}\right)-F\right)\right|_{\bar{A}_{\alpha}} .
\end{aligned}
$$

Noticing

$$
\operatorname{supp}\left(\chi_{\epsilon, \beta_{i}}\right) \cap A \subset W_{\epsilon, \beta_{i}, l_{\epsilon, \beta_{i}}} \cap A \subset U\left(A_{\beta_{i}}\right)
$$

and continuity, we get

$$
j_{\bar{A}_{\alpha}}\left((-1)^{i} \chi_{\epsilon, \beta_{1}}(x) \chi_{\epsilon, \beta_{2}}(x) \ldots \chi_{\epsilon, \beta_{i}}(x)\right) \equiv 0
$$

if $U\left(A_{\beta_{i}}\right) \cap A_{\alpha}=\varnothing$. Since $U\left(A_{\beta_{i}}\right) \cap A_{\alpha} \neq \varnothing$ implies $\bar{A}_{\alpha} \supset A_{\beta_{i}}$, we obtain

$$
\begin{aligned}
& j_{\bar{A}_{\alpha}}\left(g_{\epsilon}\right)-\left.F\right|_{\bar{A}_{\alpha}}= \\
& \quad=\left.\sum_{i=1}^{\# \Lambda} \sum_{\beta_{1} \prec \beta_{2} \prec \cdots \prec \beta_{i} \preceq A_{\alpha}} j_{\bar{A}_{\alpha}}\left((-1)^{i} \chi_{\epsilon, \beta_{1}}(x) \chi_{\epsilon, \beta_{2}}(x) \ldots \chi_{\epsilon, \beta_{i}}(x)\right)\left(j_{A}\left(g_{\beta_{i}}\right)-F\right)\right|_{\bar{A}_{\alpha}} .
\end{aligned}
$$

Now, let $\beta_{1}, \ldots, \beta_{i}$ be such that $\beta_{1} \prec \beta_{2} \prec \cdots \prec \beta_{i} \preceq A_{\alpha}$. As $l_{\epsilon, \beta_{1}}>l_{\epsilon, \beta_{2}}>$ $\cdots>l_{\epsilon, \beta_{i}}$, we have that, for any $h$,

$$
\left\|j_{A}\left((-1)^{i} \chi_{\epsilon, \beta_{1}}(x) \chi_{\epsilon, \beta_{2}}(x) \ldots \chi_{\epsilon, \beta_{i}}(x)\right)\right\|_{A, s, \# \Lambda h} \leq C_{h}^{\# \Lambda} \exp \left(\# \Lambda \varphi\left(l_{\epsilon, \beta_{i}}^{-\sigma}\right)\right) .
$$

We also have, for any $h>0$,

$$
\left\|j_{A}\left(g_{\beta_{i}}\right)-F\right\|_{B\left(A_{\beta_{i}}, l_{\epsilon, \beta_{i}}\right), s, h} \leq C_{h}^{\prime} \exp \left(-\psi\left(l_{\epsilon, \beta_{i}}^{-\sigma}\right)\right) .
$$

Since we have $W_{\epsilon, \beta_{i}, l_{\epsilon, \beta_{i}}} \cap A \subset W_{\epsilon, \beta_{i}, l_{\epsilon, \beta_{i}}} \cap U\left(A_{\beta_{i}}\right) \subset B\left(A_{\beta_{i}}, l_{\epsilon, \beta_{i}}\right)$, we get

$$
\operatorname{supp}\left(\chi_{\epsilon, \beta_{i}}\right) \cap \bar{A}_{\alpha} \subset W_{\epsilon, \beta_{i}, l_{\epsilon, \beta_{i}}} \cap \bar{A}_{\alpha} \subset B\left(A_{\beta_{i}}, l_{\epsilon, \beta_{i}}\right) .
$$

Hence, for any $h>0$, we obtained

$$
\begin{gathered}
\left\|\left.j_{\bar{A}_{\alpha}}\left((-1)^{i} \chi_{\epsilon, \beta_{1}}(x) \chi_{\epsilon, \beta_{2}}(x) \ldots \chi_{\epsilon, \beta_{i}}(x)\right)\left(j_{A}\left(g_{\beta_{i}}\right)-F\right)\right|_{\bar{A}_{\alpha}}\right\|_{\bar{A}_{\alpha}, s,(\# \Lambda+1) h} \\
\leq\left\|j_{\bar{A}_{\alpha}}\left((-1)^{i} \chi_{\epsilon, \beta_{1}}(x) \chi_{\epsilon, \beta_{2}}(x) \ldots \chi_{\epsilon, \beta_{i}}(x)\right)\right\|_{B\left(A_{\beta_{i}}, l_{\epsilon, \beta_{i}}\right) \cap \bar{A}_{\alpha}, s, \# \Lambda h} \\
\cdot\left\|\left.\left(j_{A}\left(g_{\beta_{i}}\right)-F\right)\right|_{\bar{A}_{\alpha}}\right\|_{B\left(A_{\beta_{i}}, l_{\epsilon, \beta_{i}}\right) \cap \bar{A}_{\alpha}, s, h} \\
\leq C_{h}^{\# \Lambda} C_{h}^{\prime} \exp \left(\# \Lambda \varphi\left(l_{\epsilon, \beta_{i}}^{-\sigma}\right)-\psi\left(l_{\epsilon, \beta_{i}}^{-\sigma}\right)\right) .
\end{gathered}
$$

In the end, if we take a super growth indicator $\varphi(t)$ such that

$$
\varphi(t) \ll \frac{1}{2 \# \Lambda} \psi(t),
$$


then we obtain

$$
\begin{aligned}
& \left\|\left.j_{\bar{A}_{\alpha}}\left((-1)^{i} \chi_{\epsilon, \beta_{1}}(x) \chi_{\epsilon, \beta_{2}}(x) \ldots \chi_{\epsilon, \beta_{i}}(x)\right)\left(j_{A}\left(g_{\beta_{i}}\right)-F\right)\right|_{\bar{A}_{\alpha}}\right\|_{\bar{A}_{\alpha}, s,(\# \Lambda+1) h} \\
& \leq C C_{h}^{\# \Lambda} C_{h}^{\prime} \exp \left(-\frac{1}{2} \psi\left(l_{\epsilon, \beta_{i}}^{-\sigma}\right)\right) \rightarrow 0 \quad(\epsilon \rightarrow 0)
\end{aligned}
$$

for any $h>0$.

\section{BIBLIOGRAPHY}

[1] E. Bierstone \& P. D. Milman - "Semianalytic and subanalytic sets", Publ. Math. I.H.É.S. 67 (1988), p. 5-42.

[2] N. HondA - "On the reconstruction theorem of holonomic modules in the Gevrey classes", Publ. Res. Inst. Math. Sci. 27 (1991), p. 923-943.

[3] J.-M. Kantor - "Classes non-quasi analytiques et décomposition des supports des ultradistributions", An. Acad. Brasil. Ci. 44 (1972), p. 171180.

[4] M. Kashiwara - "The Riemann-Hilbert problem for holonomic systems", Publ. Res. Inst. Math. Sci. 20 (1984), p. 319-365.

[5] M. Kashiwara \& P. Schapira - Sheaves on manifolds, Grund. Math. Wiss., vol. 292, Springer, 1990.

[6] _ "Moderate and formal cohomology associated with constructible sheaves", Mém. Soc. Math. France (N.S.) 64 (1996), p. 76.

[7] _ "Ind-sheaves", Astérisque 271 (2001), p. 136.

[8] H. Komatsu - "Ultradistributions. I. Structure theorems and a characterization", J. Fac. Sci. Univ. Tokyo Sect. IA Math. 20 (1973), p. 25-105.

[9] _ "Ultradistributions. II. The kernel theorem and ultradistributions with support in a submanifold", J. Fac. Sci. Univ. Tokyo Sect. IA Math. 24 (1977), p. 607-628.

[10] K. KURDYKA - "On a subanalytic stratification satisfying a Whitney property with exponent 1", in Real algebraic geometry (Rennes, 1991), Lecture Notes in Math., vol. 1524, Springer, 1992, p. 316-322.

[11] A. LAMBERT - "Quelques théorèmes de décomposition des ultradistributions", Ann. Inst. Fourier (Grenoble) 29 (1979), p. 57-100.

[12] S. ŁojASIEwiCZ - "Sur le problème de la division", Studia Math. 18 (1959), p. $87-136$.

[13] B. Malgrange - Équations différentielles à coefficients polynomiaux, Progress in Math., vol. 96, Birkhäuser, 1991.

[14] G. Morando - "Tempered holomorphic solutions of D-modules on curves and formal invariants", Ann. Inst. Fourier (Grenoble) 59 (2009), p. 16111639 . 
[15] L. Prelli - "Microlocalization of subanalytic sheaves", C. R. Math. Acad. Sci. Paris 345 (2007), p. 127-132.

[16] C. Roumieu - "Ultra-distributions définies sur $R^{n}$ et sur certaines classes de variétés différentiables", J. Analyse Math. 10 (1962/1963), p. 153-192.

[17] P. SchapIRA - "Sur les ultra-distributions", Ann. Sci. École Norm. Sup. 1 (1968), p. 395-415.

[18] H. WHITNEY - "Functions differentiable on the boundaries of regions", Ann. Math. 35 (1934), p. 482-485.

[19] S. YAMAZAKI - "Remark on division theorem of ultradistributions by Fuchsian differential operator", in Algebraic analysis and the exact WKB analysis for systems of differential equations, RIMS Kôkyûroku Bessatsu, B5, Res. Inst. Math. Sci. (RIMS), Kyoto, 2008, p. 209-223. 